\title{
A Molecular Pathway for Arterial-Specific Association of Vascular Smooth Muscle Cells
}

Amber N. Stratman1,4, Margaret C. Burns1*, Olivia M. Farrelly1*, Andrew E. Davis1, Wenling Li2, Van N. Pham1, Daniel Castranova1, Joseph J. Yano1, Lauren M. Goddard3, Oliver Nguyen1, Marina Venero Galanternik1, Timothy J. Bolan1, Mark L. Kahn3, Yohsuke Mukouyama2, and Brant M. Weinstein1,+

1. Division of Developmental Biology, National Institute of Child Health and Human Development, National Institutes of Health, Bethesda, MD, 20892

2. Cell and Developmental Biology Center, National Heart, Lung, and Blood Institute, National Institutes of Health, Bethesda, MD, 20892

3. Department of Medicine and Cardiovascular Institute, University of Pennsylvania, 3400

Civic Center Boulevard, Philadelphia, PA 19104

4. Department of Cell Biology and Physiology, Washington University School of Medicine,

St. Louis, MO 63110

+Correspondence: Division of Developmental Biology, National Institute of Child Health and Human Development, National Institutes of Health, Bethesda, MD 20892

e-mail: flyingfish2@nih.gov

*These authors contributed equally

Key Words: vascular smooth muscle, pericyte, zebrafish, PDGFR signaling, cxcr4 signaling, Klf2, artery stabilization 


\begin{abstract}
The preferential accumulation of vascular smooth muscle cells on arteries versus veins during early development is a well-described phenomenon, but the molecular pathways underlying this polarization are not well understood. During zebrafish embryogenesis the cxcr4a receptor (mammalian CXCR4) and its ligand $c x c l 12 b$ (mammalian CXCL12) are both preferentially expressed on arteries at time points consistent with the arrival and differentiation of the first vascular smooth muscle cells (vSMCs). We show that autocrine cxcl12b/cxcr4 activity leads to increased production of the vSMC chemoattractant ligand $p d g f b$ by endothelial cells in vitro and increased expression of $p d g f b$ by arteries in vivo. Additionally, we demonstrate that expression of the well-characterized blood flow-regulated transcription factor $k l f 2 a$ in primitive veins negatively regulates $c x c r 4 / c x c l 12$ and $p d g f b$ expression, restricting vSMC recruitment to the arterial vasculature. Together, this signaling axis leads to the differential acquisition of smooth muscle cells at sites where $k l f 2 a$ expression is low and both $c x c r 4 a$ and $p d g f b$ are co-expressed, i.e. arteries during early development.
\end{abstract}




\section{INTRODUCTION}

Endothelial cells (ECs) and mural cells are the main cellular constituents required for assembly of the arterial vascular wall. ECs form a single cell-thick, lumenized tube that is in direct contact with blood cells, immune cells, and hemodynamic forces. The endothelium is then surrounded by mural cells, including vascular smooth muscle cells (vSMCs) and pericytesperivascular cell populations that promote long-term vessel stabilization and regulate vascular tone. vSMCs are largely associated with large caliber vessels, in particular arteries, while pericytes are associated with smaller vessels such as those in capillary beds. Arterial associated vSMCs provide tensile strength to the vascular wall by countering blood flow forces coming out of the heart, promoting maintenance and assembly of the vascular basement membrane, and helping regulate blood pressure (Bergers and Song, 2005; Hall-Glenn et al., 2012; Hedin et al., 1999; Heickendorff, 1989; Le et al., 2011; Lee et al., 2019; Leveen et al., 1994; Lilly, 2014; Lindahl et al., 1997; Osei-Owusu et al., 2014; Santoro et al., 2009; Scheppke et al., 2012; Stratman and Davis, 2012; Stratman et al., 2009; Stratman et al., 2017). Although vSMCs are critical modulators of arterial vasculature function, very little is known about the molecular cues that direct their preferential association with arteries rather than veins during early development. This preference has largely been thought to be mediated by differences in blood flow patterns, rates, and shear stresses associated with the arterial vasculature versus the venous vasculature (Ando et al., 2016; Chang et al., 2012; Lin and Lilly, 2014; Roostalu and Wong, 2018; Stratman et al., 2017). Although flow-mediated cues are likely critical, the cellular effectors mediating responses to blood flow and shear stress have yet to be fully elucidated.

The Klf2 transcription factor is one of the best-known blood flow-regulated genes, making it an attractive candidate for a role in flow-based regulation of VSMC recruitment. Klf 2 is heavily expressed by postnatal endothelial cells and immune cells, and it has been studied extensively for its connection to atherosclerosis (Arkenbout et al., 2003; Atkins and Jain, 2007; Dekker et al., 2002; Dekker et al., 2005; Goddard et al., 2017; Huddleson et al., 2004; Kuo et al., 1997; Lee et al., 2006; Methe et al., 2007; Nayak et al., 2011; van Thienen et al., 2006). In atherosclerotic disease, sites of low Klf2 expression are prone to lesion development, hinting that suppressed Klf2 mediated signaling is linked to an activated state of cells comprising the vascular wall (Davies et al., 2013; Dekker et al., 2006; Dekker et al., 2005; Gimbrone and Garcia-Cardena, 2013; Goddard et al., 2017; Komaravolu et al., 2015; Mack et al., 2009; Parmar et al., 2006). Indeed, in vitro 
models suggest that EC induction of Klf 2 could be a negative regulator of adjacent vSMC motility via promoted cellular differentiation (Dekker et al., 2006; Franzoni et al., 2019; Mack et al., 2009). Studies in mice, zebrafish, and in vitro have implicated Klf2 in angiogenesis, remodeling of the aortic outflow tract, heart formation and valve formation, and vascular stabilization during development (Adam et al., 2000; Bhattacharya et al., 2005; Chiplunkar et al., 2013; Dekker et al., 2002; Goddard et al., 2017; Kuo et al., 1997; Lee et al., 2006; Lin et al., 2010; Liu et al., 2005; Mack et al., 2009; Methe et al., 2007; Novodvorsky and Chico, 2014; Sangwung et al., 2017; Wu et al., 2008). However, the role of Klf2 in vSMC biology and function, and how it interfaces with known pathways and factors regulating vSMC recruitment and differentiation, remains largely unexplored.

Platelet Derived Growth Factor B (PDGFB) is one of the best-described factors regulating vSMC/mural cell biology. PDGFB ligand is produced by endothelial cells and it signals to mural cells via the PDGFRB receptor. A variety of studies have shown that this signaling pathway is critical for mural cell recruitment and proliferation, including the original reports demonstrating that PDGFRB knockout mice have decreased mural cell coverage of vessels and increased vessel dilation (Abramsson et al., 2007; Hellstrom et al., 1999; Leveen et al., 1994; Lindahl et al., 1997; Lindblom et al., 2003; Roostalu and Wong, 2018; Stratman and Davis, 2012; Stratman et al., 2017; Stratman et al., 2010). However, while recruitment of vSMCs is almost exclusively restricted to arteries during early development, PDGFB ligand is initially expressed on both primitive veins and arteries, before becoming largely restricted to arteries (Claxton et al., 2008; Hellstrom et al., 1999; Payne et al., 2018; Stanczuk et al., 2015), suggesting that other factors may help to suppress PDGFB expression in veins to direct vSMC recruitment to arteries.

The CXCR4 chemokine receptor and its associated ligand CXCL12 (aka SDF1a) are both expressed heavily on arteries or in tissue directly adjacent to arteries during early development (Busillo and Benovic, 2007; Bussmann et al., 2011; Cha and Weinstein, 2012; Corti et al., 2011; Fujita et al., 2011; Li et al., 2013). Chemokine signaling has been reported to have effects on vascular development, including during formation of blood vessels, formation of lymphatics, and potentially differentiation and recruitment of mural cells (Busillo and Benovic, 2007; Bussmann et al., 2011; Cha et al., 2012; Doring et al., 2014; Fujita et al., 2011; Gupta et al., 1998; Harrison et al., 2015; Li et al., 2013; Noels et al., 2014; Sebzda et al., 2008; Siekmann et al., 2009; Stratman et al., 2011). Knockout of CXCR4 in mice results in defective arterial patterning in the developing 
skin, in particular lack of alignment with nerves, with associated defects in mural cell coverage of the vasculature (the effects on large vessels were not analyzed in these studies) (Li et al., 2013). Other studies have suggested more direct effects of chemokine signaling in helping mural cells maintain their de-differentiated status, allowing for increased cellular motility, proliferation, and synthetic/matrix producing phenotype (Doring et al., 2014; Hamdan et al., 2011, 2014; Noels et al., 2014; Shi et al., 2014). CXCR4 and PDGFB are both thought to be blood flow responsive genes, suggesting potential links to Klf2 (Busillo and Benovic, 2007; Bussmann et al., 2011; Corti et al., 2011).

In this report we elucidate a molecular pathway promoting preferential association of vSMC with arterial blood vessels. We demonstrate that CXCL12/CXCR4 chemokine signaling in the arterial endothelium promotes PDGFB ligand expression, driving arterial vSMC recruitment. We also show that venous expression of KLF2 after the onset of blood flow negatively regulates CXCR4 and PDGFB production to inhibit venous recruitment of vSMC. Together, our findings highlight a molecular pathway driving arterial-specific recruitment of vSMCs, via regulated and coupled expression of chemokines and PDGFB in the vasculature. 


\section{RESULTS}

\section{Smooth muscle cells preferentially associate with developing arteries}

The presence of a thickened vascular wall with abundant vascular smooth muscle cells (vSMC) is perhaps the most clearly evident morphological feature distinguishing arteries from veins, but the molecular mechanisms responsible for preferential acquisition of vSMC by arteries remains largely unexplored. As we and others have previously reported, vSMC emerge from the medial sclerotome of the zebrafish trunk beginning at approximately $3 \mathrm{dpf}$, taking up residence around the closely juxtaposed dorsal aorta, but not around the equally closely adjacent cardinal vein (Fig. 1A; (Ando et al., 2016; Santoro et al., 2009; Stratman et al., 2017; Whitesell et al., 2014)). This differential recruitment of vSMC to the trunk dorsal aorta but not the cardinal vein is readily observed in $5 \mathrm{dpf} T g(\operatorname{tag} \ln : e G F P), T g(k d r l: m C h e r r y-C A A X)$ double-transgenic zebrafish with mCherry-positive vascular endothelium (magenta) and eGFP-positive vSMC (green) (Fig. 1B,C). To identify candidate factors that might be playing a role in guiding the dorsal aortarestricted recruitment of vSMC, we used the ZFIN gene expression database (zfin.org) to carry out an in silico survey for secreted factors differentially expressed in the dorsal aorta but not in the cardinal vein at approximately $2.5 \mathrm{dpf}$. The $\operatorname{cxcl} 12 \mathrm{~b}$ chemokine ligand (also known as SDF1a) and its receptor $\operatorname{cxc} 4 a$ were identified as promising candidates. Although, expressed in both cardinal vein and dorsal aorta at earlier stages of development (1.25 dpf, data not shown), by $2.5 \mathrm{dpf}$ when vSMC recruitment is beginning, $c x c l 12 b$ and $c x c r 4 a$ expression is strongly enriched in the dorsal aorta compared to the cardinal vein (Fig. 1D-G).

\section{Chemokine signaling regulates vSMC association with arteries}

To examine whether cxcl12b/cxcr $4 a$ chemokine signaling plays a role in dorsal aortaspecific vSMC recruitment, we used CRISPR/Cas9 technology to generate 24 bp and 7 bp deletion mutants in cxcl12b and cxcr4a, respectively (Supp. Fig. 1A,B). Homozygous cxcl12b $\triangle 24 / \Delta 24$ mutant animals show reduced numbers of vSMC associated with the dorsal aorta at $5 \mathrm{dpf}$ (Fig. 2A-C) and an increased dorsal aorta diameter (Fig. 2D), a previously-reported consequence of defects in vSMC coverage of vessels (Stratman et al., 2009; Stratman et al., 2017). Homozygous cxcr4as7/47 mutant animals also have reduced numbers of dorsal aorta-associated vSMC and increased dorsal aorta diameter (Fig. 2E-H). Deletion of the Cxcr4 gene in mice similarly results in decreased thickness of the dorsal aorta vSMC (smooth muscle actin-positive) wall (Fig. 2I-K) 
and dorsal aorta luminal enlargement (Fig. 2L) at E12.5, suggesting the role of chemokine signaling in vSMC recruitment is evolutionarily conserved. If chemokine signaling is indeed promoting vSMC recruitment to the dorsal aorta, we reasoned that forced mis-expression of $c x c l 12 b$ in the cardinal vein (where it is not normally expressed at $3 \mathrm{dpf}$ ) might result in ectopic targeting of vSMC to this vessel (Fig. 3A,B). To test this, we injected $T g(\operatorname{tag} \ln : e G F P$ ) transgenic zebrafish with a Tol 2 transgene containing the $m r c l a$ promoter driving expression of $c x c l 12 b$ and mCherry, co-translationally linked together via the $2 \mathrm{~A}$ peptide sequence (Fig. 3A,B). We have previously shown that the mrcla promoter drives robust expression in the early cardinal vein in addition to other vessels (Jung et al., 2017). Mosaic expression of cxcl12b in the cardinal vein (marked by mCherry expression) promoted ectopic cardinal vein recruitment of vSMC without affecting vSMC acquisition by the dorsal aorta (Fig. 3C-E). Together these gain- and loss-offunction experiments support the idea that dorsal aorta-restricted chemokine signaling is important for differential arterial recruitment of vSMC.

\section{PDGFB modulates vSMC association with the dorsal aorta}

We carried out additional experiments to examine whether CXCL12 acts directly as a paracrine chemoattractant for arterial vSMC or indirectly via autocrine activation of arterial endothelial CXCR4 to induce expression of some other chemoattractant factor (Fig. 3F). Using a previously described in vitro 3-D cell motility assay (Fig. 3G; (Koh et al., 2008; Stratman et al., 2011; Stratman et al., 2009)), we showed that human coronary artery smooth muscle cells (CASMCs) have little or no chemoattractant activity for CXCL12, unlike PDGFB which has a robust activity in this assay (Fig. 3H,I). PDGFB is a well-documented in vivo vSMC chemoattractant expressed by endothelial cells and required for vSMC recruitment in mice (Abramsson et al., 2007; Hellstrom et al., 1999; Leveen et al., 1994). Zebrafish $p d g f b$ is expressed weakly in both the dorsal aorta and cardinal vein at $1.5 \mathrm{dpf}$ (Fig. 4A-C), but by the time vSMC recruitment begins at $3 \mathrm{dpf}$ it shows preferential expression in the developing dorsal aorta (Fig. 4C). To examine the role of $p d g f b$ in vSMC recruitment in the zebrafish we used CRISPR/Cas9 mutagenesis to generate mutants in the $p d g f b b$ and closely related $p d g f b a$ genes (Supp. Fig. 1C). Zebrafish homozygous mutants for either $p d g f b b$ or $p d g f b a$ alone display modest reductions in vSMC recruitment to the dorsal aorta at $5 \mathrm{dpf}$, but animals homozygous for the mutations in both genes display a strong reduction in vSMC recruitment (Fig. 4D-F). Together with previous findings from our lab using expression of dominant-negative $p d g f r b$-DN to disrupt vSMC 
recruitment (Stratman et al., 2017), these results suggest that, as noted in mice, PDGFB signaling serves as an important vSMC chemoattractant in the zebrafish.

\section{Chemokine signaling regulates expression of PDGFB}

To test the possibility that CXCL12/CXCR4 signaling acts indirectly on vSMC motility via autocrine up-regulation of PDGFB in arterial endothelium (Fig. 4G), we examined whether chemokine signaling regulates PDGFB transcript and/or protein levels in HUVECs in vitro (Fig. 5A-E). Application of exogenous recombinant CXCL12 ligand to HUVECs in culture results in increased levels of PDGFB transcript (Fig. 5A) and PDGFB protein (Fig. 5B). Conversely, siRNA knockdown of endogenous CXCR4 or CXCL12 in cultured HUVECs results in decreased PDGFB transcript levels (Fig. 5C) and decreased PDGFB protein levels (Fig. 5D,E). To determine whether Cxcr4 is also required for Pdgfb expression in the endothelium in vivo, we used immunostaining to examine Pdgfb expression in the developing arteries of Cxcr4 knockout mice. Compared to their heterozygous siblings, Cxcr4-/- homozygous null mice showed a strong reduction in arterial expression of Pdgfb ligand, as well as reduced vSMC coverage as assessed by Sm22+ staining at E12.5 (Fig. 5F,G). Conversely, overexpression of $c x c l 12 b$ ligand in zebrafish larvae via injection of $c x c l 12 b$ RNA resulted in an increase in $p d g f b$ transcript (Fig. 5H) and protein levels (Fig. 5I) as assessed by in situ hybridization and western blotting respectively. Together, these results suggest that autocrine CXCL12/CXCR4 chemokine signaling up-regulates PDGFB ligand in endothelial cells to promote recruitment of vSMC (Fig. 5J).

\section{KLF2 regulates vSMC recruitment upstream from chemokine signaling}

We next sought to understand what might be responsible for preferentially restricting CXCL12/CXCR4 signaling to the dorsal aorta rather than the cardinal vein. Our primary candidate gene of interest was Klf2 - a well documented blood flow-regulated transcription factor, with reported links to chemokine signaling. In the zebrafish, $k l f 2 a$ begins to be expressed in the cardinal vein just after the onset of blood flow between 24 and $32 \mathrm{hpf}$, and it remains expressed predominantly by veins through at least 72 hpf (Fig. 6A). Preferential expression of Klf 2 in primitive veins is also noted in E9.5 mice (Fig. 6B), with Klf2 expression enriched in both the cardinal vein and the vitelline vein as compared to the dorsal aorta. These results, and previous reports that KLF2 can negatively regulate chemokine signaling, suggested to us that KLF2 expression in primitive veins might act as a "STOP" signal to block CXCL12/CXCR4 and PDGFB 
expression in the cardinal vein, thus restricting vSMC recruitment to the dorsal aorta. If this were the case, we predicted removing KLF2 would increase chemokine signaling and PDGFB expression to promote ectopic venous recruitment of vSMC.

To test this idea, we used siRNA knockdown to suppress KLF2 in HUVECs in vitro and genetic mutants to "knock out" klf2a in the zebrafish in vivo. Suppressing KLF2 in HUVECs in vitro led to upregulation of CXCR4 and PDGFB transcript and protein levels (Fig. 6D-H). To examine the consequences of reduced KLF2 in the endothelium in vivo, we used CRISPR/Cas9 mutagenesis to generate an 8 bp deletion mutant in zebrafish klf2a (Supp. Fig. 1D). Although there was no change in the number of vSMCs associated with the dorsal aorta in homozygous klf $2 a \Delta 8 / \Delta 8$ mutants (Fig. 6I,L,M), there was a clear increase in the number of vSMCs associated with the cardinal vein (Fig. 6J,L,M). This increase was accompanied by a modest decrease in the diameter of the cardinal vein without any change in the dorsal aorta (Fig. 6K), consistent with the role of vSMC in regulating vessel diameter. Together, these results suggest that expression of KLF2 prevents chemokine/PDGF-mediated association of vSMC to primitive veins. 


\section{DISCUSSION}

\section{A molecular pathway for vSMC recruitment}

The preferential association of vascular smooth muscle cells with arteries has been appreciated for centuries, but the molecular mechanisms underlying this preference have not been fully elucidated. We have uncovered a molecular pathway that promotes association of vSMCs with developing arteries and reduces vSMC association with veins (Fig. 7). The known blood flow-regulated transcription factor KLF2 helps demarcate and maintain a pro-vSMC state in the endothelium. Sustained expression of KLF2 (zebrafish $k l f 2 a$ ) in primitive venous endothelium during early development represses expression of $\operatorname{cxcl12b}$ and $\operatorname{cxc} 4 a$ and autocrine/juxtacrine endothelial chemokine signaling, thereby reducing chemokine signaling-promoted production of $p d g f b$ and venous recruitment of vSMC. In contrast, down-regulation of $k l f 2 a$ in the early arterial endothelium permits expression of $c x c l 12 b$ and $c x c r 4 a$, promoting production of arterial $p d g f b$ and arterial recruitment of vSMC (Fig. 7). As predicted by this model, either artificially suppressing $k l f 2 a$ expression or mis-expressing $c x c l 12 b$ in venous endothelium promotes ectopic venous recruitment of vSMC (Fig. 3A-E, Fig. 6J).

\section{The role of blood flow in vSMC recruitment and differentiation}

Together, our findings and previous reports describing flow-mediated regulation of KLF2 suggest that changes in blood flow velocity, force, or directionality influence stabilization of the arterial blood vessel wall by modulating the expression of this key transcription factor. In the zebrafish trunk, $k l f 2 a$ expression is initially equivalently low in the endothelial cords that will give rise to the dorsal aorta (DA) and cardinal vein $(\mathrm{CV})$, but as these vessels lumenize and circulation begins, the expression of $k l f 2 a$ is strengthened in the CV. Since flow begins in the DA and CV at the same time during early development, why do these two vessels show such different $k l f 2 a$ expression, and how could flow be playing a role in this difference? Although further work will be needed to definitively answer this question, the type of flow that each of these vessels experience is likely playing a critical role in their differential expression of $k l f 2 a$. There is relatively even, constant laminar flow through the $\mathrm{CV}$ at these early developmental stages, while the DA experiences a much more pulsatile flow regime. Pulsatile flow results in cyclical changes in velocity and shear, and in the circumferential "stretch" experienced by the endothelial cells in the DA, that are not experienced by $\mathrm{CV}$ endothelial cells. Indeed, our previously published findings 
show a high degree of pulsatility and cyclical change in diameter of the DA with blood flow pulsation during early development (Stratman et al., 2017). In vitro and in vivo studies have shown that KLF2 expression is differentially regulated by different types of flow regimes - typically showing increased expression by laminar flow and reduced expression associated turbulent, nonlaminar flow (Goddard et al., 2017; Lee et al., 2006; Lin et al., 2010; Methe et al., 2007; Nayak et al., 2011; Parmar et al., 2006).

Although we show that developing primitive arteries show reduced KLF2 expression compared to primitive veins in both zebrafish and mice, the endothelium of mature arteries expresses high levels of KLF2 (Goddard et al., 2017; Methe et al., 2007; Nayak et al., 2011; Parmar et al., 2006). How can the latter results be reconciled with our model (Fig. 7) in which "arterial" blood flow reduces KLF2 expression and the presence of KLF2 suppresses arterial-specific recruitment of vSMC? These results make sense if the expression of KLF2 is responsive to endothelial cell stretch/strain. Primitive arteries initially only consist of an endothelial layer without encircling smooth muscle layers, resulting in high levels of endothelial stretch and strain in response to pulsatile flow, as noted above. Gradual acquisition of vSMCs and assembly of vascular basement membrane layers creates resistance to the cyclical changes in vessel diameter with blood flow pulsation, reducing the "stretch" experienced by endothelial cells. Again, our previously published results show reduced vessel wall movements and pulsatile blood flowinduced changes in diameter as the DA acquires vSMC (Stratman et al., 2017). Endothelial recruitment of VSMC is required for formation of a vascular basement membrane, and this is also critical for reducing vessel distensibility, and thus endothelial cell stretch/strain (Stratman and Davis, 2012; Stratman et al., 2009; Stratman et al., 2017; Stratman et al., 2010).

Together, these data point to dynamic, flow-regulated expression of KLF2 as a mechanism not only for promoting arterial-specific recruitment of vSMC, but also for self-limiting and selftuning the extent of vSMC acquisition to match the specific hemodynamic requirements of different regions of the arterial vascular tree. As developing arteries acquire vSMC and vSMCpromoted basement membrane, reduced vessel compliance/increased stiffness would be predicted to result in increased KLF2 expression, creating a "stop signal" to halt further vSMC recruitment and promote vSMC differentiation and quiescence (Adam et al., 2000; Liu et al., 2005; Sebzda et al., 2008). In larger and/or more proximal arteries experiencing more dramatic cyclic flux in blood pressure, the "stop signal" might be expected to come later, after acquisition of a greater amount 
of vSMC and matrix and formation of a thicker and more robust vessel wall. Thus, this mechanism would ensure that the capacity of the vascular wall to resist stretch matches the flow regime vessels experience. Although this seems like a very attractive mechanism to allow arteries to "selfregulate" vSMC acquisition based on intrinsic flow dynamics, additional experimental studies will of course be needed to further substantiate that this is indeed an important determinant of the extent of vascular wall assembly.

\section{Developmental pathways reactivated in disease?}

A number of disease states have known links to altered blood flow sensing and/or are correlated with altered Klf2 expression profiles, including atherosclerosis and Cerebral Cavernous Malformations (CCMs), and it seems possible that the KLF2-CXCL12/CXCR4-PDGFB molecular pathway that we have identified for arterial vSMC acquisition during development may also play critical roles in vascular pathologies. As noted in our introduction, sites of low Klf2 expression are correlated with atherosclerotic lesion formation (Atkins and Jain, 2007; Komaravolu et al., 2015; Lee et al., 2006; Parmar et al., 2006). The experimental data from our study showing that low Klf2 expression correlates with high chemokine and high PDGFB expression suggests that suppressed Klf2 could be promoting atherogenesis at least in part by promoting chemokine/PDGFB-stimulated vSMCs proliferation and reactivated motility (Adam et al., 2000; Dandre and Owens, 2004; Doring et al., 2014; Liu et al., 2005; Noels et al., 2014; Owens et al., 2004; Sebzda et al., 2008; Sen-Banerjee et al., 2005). Altered chemokine signaling has already been implicated in coronary artery disease, mobilization of progenitor cells in ischemia induced remodeling/repair, and atherosclerosis (Busillo and Benovic, 2007; Doring et al., 2014; Gupta et al., 1998; Noels et al., 2014; Sebzda et al., 2008; Shi et al., 2014). Upregulated chemokine signaling can lead to increased smooth muscle cell proliferation and chemotaxis, and it is known that vSMCs actively de-differentiate and invade atherosclerotic plaques (Doring et al., 2014; Noels et al., 2014). Our results would suggest that the effects of chemokine signaling on the motility and proliferation of vSMCs in these pathological contexts may be indirect, through upregulation of PDGFB. Other disease models also offer some support for this idea.

CCM pathology, on the other hand, is associated with high Klf2/4 expression ( $\mathrm{Li}$ et al., 2019; Renz et al., 2015; Zhou et al., 2016). CCM is a vascular malformation disorder particularly localized to intersection points between capillaries and veins that leads to the formation of large dilated vessels (Castro et al., 2019; Li et al., 2019; Renz et al., 2015; Zhou et al., 2016). These 
dilated vessels are prone to rupture, leading to trauma, tissue damage, and ischemia. Formation of CCM lesions has been linked to mutations in one of three different CCM proteins, that appear to have their deleterious effects at least in part via a Cdc42-MEKK3-MEK5-ERK5-KLF2/4 signaling cascade within the endothelium (Castro et al., 2019; Komaravolu et al., 2015; Li et al., 2019; Parmar et al., 2006; Zhou et al., 2016). Vessels in CCM lesions have low numbers of associated vSMC/mural cells, consistent with the idea that high Klf2 levels serve as a "stop" signal for vSMC recruitment. It will be interesting to further determine whether vSMC recruitment and the KLF2CXCL12/CXCR4-PDGFB pathway we have identified are involved in a causative way in lesion formation, or only secondarily affected as a result and/or part of developing CCM lesion pathology.

\section{Concluding Remarks}

We describe a signaling pathway that promotes association of vSMCs with the arterial vasculature and restrains association with the venous vasculature during development. This pathway links blood flow, through the transcription factor Klf2, to chemokine signaling, PDGFB production, and ultimately arterial endothelium-directed motility of vSMCs. In addition to its important role in arterial/venous development, this signaling cascade may also be involved in vascular pathologies that alter vSMC acquisition or function. 


\section{METHODS}

\section{Zebrafish Methods and Zebrafish Transgenic Lines}

Zebrafish (Danio rerio) embryos were raised and maintained as described (Kimmel et al., 1995; Westerfield, 1995). Zebrafish husbandry and research protocols were reviewed and approved by the NICHD Animal Care and Use Committee. Zebrafish transgenic lines Tg $(k d r l: m C h e r r y-C A A X)$ y171; $\mathrm{Tg}($ tagln:egfp $)$ p151 are previously published. New CRISPR/Cas9 mutant alleles generated for

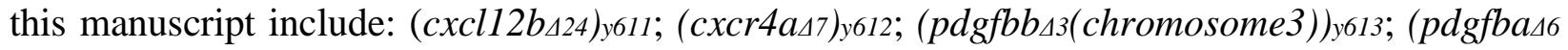
(chromosome22) $)_{y 614} ;($ klf2as8)y616

\section{Reagents}

Antibodies for immunostaining and western blot analysis include: Tubulin (Sigma, \#T61991:10,000 dilution); PECAM-1 (CD31) (BD Pharmingen; \#553370- 1:300 dilution); alpha-sma-cy3 (Sigma; \#c-6198; 1:500 dilution); Sm22 (GeneTex; \#GTX101608; 1:300 dilution); PDGFB (SantaCruz; \# sc-365805; 1:500 dilution); CXCR4 (Sigma, \#SAB3500383); CXCL12 (R\&D Systems, \#AF-310-NA). WISH probes utilized include $k l f 2 a$ (Corti et al., 2011), pdgfb (Wiens et al., 2010), cxcr4a (Cha et al., 2012; Fujita et al., 2011), and cxcl12b (Primers FW:GAGCTCT GGACACTCGCTGT; RV:TACTGCTGAAGCCATTTGGTC)

\section{Imaging and Microscopy}

Fluorescent images were collected utilizing either the Leica SP5 II or Nikon Yokogawa CSU-W1 spinning disk confocal microscope at 5dpf at 20x magnification. Embryos were immobilized in buffered MS-222 and embedded in 0.8\% low melting point agarose. All images were acquired, data quantified and analyzed blindly, and then embryos genotyped.

\section{Endothelial Cell Culture and 3D Assays}

Human umbilical vein endothelial cells (HUVEC, Lonza) were cultured in bovine hypothalamus extract, $0.01 \%$ Heparin and 20\% FBS in M199 base media (Gibco) on 1mg/ml gelatin coated tissue culture flasks. HUVECs were used from passages 3-6.

Human coronary artery smooth muscle cells (PASMC, Lonza) were cultured in 10\% FBS in Advanced DMEM base media (Gibco) on $1 \mathrm{mg} / \mathrm{ml}$ gelatin coated tissue culture flasks. PASMCs were used from passages 3-8.

3-dimensional (3D) collagen type I in vitro assays were done essentially as described ((Stratman et al., 2009)), utilizing $2.5 \mathrm{mg} / \mathrm{mL}$ collagen type I (BD Biosciences, Acid Extracted) gels including CXCL12 (R\&D Systems, \#350-NS/CF) or PDGFB (R\&D Systems, \#220-BB/CF). PASMCs were seeded on the collagen gel at 40,000 cells per well density. Culture media for the assays contained ascorbic acid, FGF (R\&D Systems, \#233-FB-025/CF), and IGF-II (R\&D Systems, \#292-G2-250). Assays were fixed in 2\% paraformaldehyde (PFA) at 2 days and processed for future analysis.

\section{CRISPR/Cas9 Generation of Zebrafish Mutants}


Mutations in the zebrafish $c x c l 12 b, c x c r 4 a, p d g f b b, p d g f b a$, and $k l f 2 a$ genes were generated using the CRISPR/Cas9 system. The following guide RNAs were transcribed in vitro using the T7 mMessage Machine ${ }^{\circledR}$ Kit (Ambion), and injected at a dose of $150 \mathrm{pg} / \mathrm{nl}$ per embryo:

cxcl12b_24/424:

TAATACGACTCACTATAGGAGCCCAGAGACTGACGGTGTTTTAGAGCTAGAA

cxcr4as7/47:

TAATACGACTCACTATAGGACATCGGAGCCAACTTTGGTTTTAGAGCTAGAAATAGC AAG

$p d g f b b$ :

TAATACGACTCACTATAGGCTGTGGTTGAGTTGGTGAGTTTTAGAGCTAGAA

pdgfba:

TAATACGACTCACTATAGGACCCTCTTCCTCCATCTCGTTTTAGAGCTAGAA klf2a $48 / \Delta 8$ :

TAATACGACTCACTATAGGTCCGTAACTATCCATGCAGTTTTAGAGCTAGAAATAGC AAG

pT3TS-nCas9 (Addgene) was transcribed using MEGA Script T7 kit (Invitrogen/Ambion), and injected at a dose of $300 \mathrm{pg} / \mathrm{nl}$ per embryo. Embryos were injected at the single cell stage, screened for cutting efficiency and grown on system. F1 generations were analyzed for mutations, and pairs crossed for analysis in the F2 and beyond generations.

\section{Genotyping of Zebrafish Mutants}

Mutants were genotyped using the following primers:

cxcl12b 424 :

FW: TGTAAAACGACGGCCAGTGTATCACTTATATTCTCAAC

RV: GTGTCTTCACTCGCTCTTGGCATGGATAGC

cxcr4as7:

FW: TGTAAAACGACGGCCAGTCAGCACATCGTCTTTGAAGATGATTTATC

RV: GTGTCTTGGCAGAGTGAGCACAAACAGAAGG

pdgfb 44 (chromosome 3):

FW: TGTAAAACGACGGCCAGTGATTGTTTGATTAATAAGGAC

RV: GTGTCTTCTACAACATGTGACAAATTC

pdgfa $\Delta 8$ or $\Delta 20$ (chromosome 22 ):

FW: TGTAAAACGACGGCCAGTAGGTGTTGTTTTGTTCAGGACC

RV: GTGTCTTTGGTATGGGATCAGCTTTACCT

klf2ass:

FW: TGTAAAACGACGGCCAGTGACATTGACACCTACTGC

RV: GTGTCTTGAGTCATGCTGCCTGCTCC

Universal Primer: FAM-M13: 5 -/56-FAM/ TGTAAAACGACGGCCAGT -3`

\section{ABI 3130xl Fragment Analyzer Protocol}


PCR protocol with AmpliTaq Gold DNA Polymerase 1x (10ul) Rxn: 1ul 10x PCR Gold Buffer; 0.5ul MgCl2 25mM; 1ul 0.5mM ABI Fwd primer; 1ul 1mM ABI Rev primer; 0.2ul 10mM FAMM13 primer; 0.1ul dNTP Master Mix; 0.1ul TaqGold polymerase; 1ul of 1:10 diluted crude gDNA; 5.1ul H20

TaqGold PCR Program: 95。C 10min' 95。C 30sec; 58॰C 30sec; 72。C 30sec (1 min/kb); GoTo Step $2 \mathrm{x} 34 ; 72 \mathrm{oC} 10 \mathrm{~min} ; 15 \mathrm{oC}$ Hold; Run on $\mathrm{ABI}$ immediately or store at $4{ }_{\mathrm{o}} \mathrm{C}$ in the dark for 24 hours $\max$.

ABI 3130xl Plate set-up: HiDi Formamide/ROX master mix- 0.2ul ROX400HD; 9.8ul HiDi Formamide; add 10ul of master mix to each ABI plate sample well; add 2ul of fluorescent PCR product; cap wells and denature at $95 \mathrm{oC}$ for $5 \mathrm{~min}$; uncap all wells and replace with $\mathrm{ABI}$ plate septa to run on the 3130xl. Follow manufacturer directions to utilize the ABI 3130xl.

\section{Mouse Lines, Breeding, and Genotyping}

Cxcr4 Knockout Mice: Cxcr4 knockout mutants: We initially generated $\mathrm{Cxcr} 4+$ /- heterozygous mice from the breeding of Cxcr4-flox mice (Jax\# 008767) with E2a-Cre mice (Jax\#003724). Cxcr4-- homozygous embryos were produced by mating Cxcr4+/- heterozygous mice. Offspring were genotyped by genomic PCR using primers that specifically detect $C x c r 4$ allele and $C x c r 4$ null allele.

Cxcr4 allele (347bp):

FW: CAC TAC GCA TGA CTC GAA ATG

RV: GTG TGC GGT GGT ATC CAG C

Cxcr4 null allele (190bp)

FW: CAC TAC GCA TGA CTC GAA ATG

RV: CCT CGG AAT GAA GAG ATT ATG C

Klf2-GFP mouse was published previously (Weinreich et al., 2009), and is a knockin allele where the GFP is fused to the N-terminus of KLF2. GFP signal was amplified using a GFP antibody (Goat anti-GFP, Abcam, CAT\#ab6673 1:250) and the endothelium labeled with PECAM-1 antibody (Dianova, CAT\# DIA-310 1:200).

\section{Immunostaining and Western blot analysis}

Tissue sections were immunostained following the same basic protocol: 1) 30 min RT incubation in Tris-Glycine; 2) $1 \mathrm{hr}$ RT incubation +/- permeabilization with $0.01 \%$ TritonX-100; 3) $2 \mathrm{hr}$ RT incubation in blocking solution (5\% Sheep Serum, 1\% Roche Blocking Buffer in PBST); 4) 1hr at RT - overnight $4 \circ \mathrm{C}$ incubation with 1:1000 primary antibody unless otherwise noted; 5) wash with PBST; 6) 2-3hr RT incubation with 1:2000 secondary antibody in 5\% Sheep Serum, 1\% Roche Blocking Buffer in PBST; 7) wash with PBST and imaging analysis. Quantification of immunostaining intensity was performed by ImageJ analysis software. Images were acquired using a Leica SP5 II confocal microscope or Nikon Yokogawa CSU-W1 spinning disk confocal microscope. All images were acquired at the same intensity, step size and image resolution for analysis. The images were analyzed using ImageJ (Schindelin et al., 2012). Data is reported as the percent average intensity per region of interest size from a minimum of 3 images from 3 independent experiments/animals \pm s.e.m. 
Zebrafish samples for Western blot analysis were deyolked and directly lysed in 2x Laemmli Sample Buffer containing 5\% b-ME and a PhosSTOP tablet (Roche), 10 ul per embryo unless otherwise indicated. HUVEC cells for Western blot analysis were lysed directly in 2x Laemmli Sample Buffer containing 5\% b-ME and a PhosSTOP tablet (Roche), 500ul per T-25 culture flask.

Secondary Digital-HRP-conjugated antibodies were purchased from Kindle Bioscience and used at 1:1000 in 5\% milk. Primary antibodies are described in the 'Reagents' section and used at 1:1000 unless otherwise noted. Images were acquired using the Kindle Bioscience KwikQuant Imager and 1-Shot Digital ECL. Quantification of relative band density was performed using ImageJ software. Data is reported as the percent average density from a minimum of 2-3 blots from at least 2 independent experiments \pm s.e.m.

\section{qPCR and RNA Extraction}

Zebrafish embryos or HUVECs were collected at the indicated time points in TRIZOL and RNA purified using a double chloroform extraction protocol. cDNA was generated using BioRads IScript cDNA synthesis kit from 500ng of RNA. TaqMan qPCR protocols were utilized to generate relative expression data, and analysis run using the FAM channel of a 96 well BioRad CFX qPCR machine. Primer product numbers are as follow:

PDGFB (human): Hs00966522_m1

CXCL12 (human): Hs03676656_mH

KLF2 (human): Hs00360439_g1

CXCR4 (human): Hs00607978_s1

Ef1a (human): Hs00265885_g1

GAPDH (human): Hs02758991_g1

\section{Statistics}

Statistical analysis of data was done using Microsoft Excel. Statistical significance was set at a minimum of $\mathrm{p} \leq 0.05$ and is indicated in individual figures. Student's t-tests were used when analyzing 2 groups within a single experiment. Bar graphs were generated with Plotly.

\section{Study Approval}

Zebrafish husbandry and research protocols were reviewed and approved by the NICHD Animal Care and Use Committee at the National Institutes of Health. All animal studies were carried out according to NIH-approved protocols, in compliance with the Guide for the Care and use of Laboratory Animals.

\section{Author Contributions}

ANS, OMF, MCB, AED, WL, VNP, DC, ON, JY, TJB, LG, and MVG performed experiments; ANS, OMF, MCB, AED, WL, VNP, DC, ON, JY, LG, MVG, TJB, MK, YSM, and BMW analyzed results and made the figures; ANS, OMF, MCB, AED, WL, VNP, DC, JY, MK, YSM, and BMW designed the research and wrote the paper. 
Conflict-of-interest disclosure: The authors declare no competing financial interests.

Correspondence: Brant M. Weinstein, Department of Developmental Biology, National Institute of Child Health and Human Development, National Institutes of Health, 6 Center Dr. Bethesda, MD 20892; e-mail: flyingfish2@nih.gov

\section{Acknowledgements}

The authors would like to thank members of the Weinstein laboratory for their critical comments on this manuscript. This work was supported by the intramural program of the Eunice Kennedy Shriver National Institute of Child Health and Human Development, National Institutes of Health (ZIA-HD001011 and ZIA-HD008915, to BMW); K99/R00 Pathway to Independence Award (NHLBI-4R00HL125683-02, to ANS); the Intramural Research Program of the National Heart, Lung, and Blood Institute, National Institutes of Health (ZIA HL005702-14 to YM). 


\section{Figure Legends}

Figure 1. vSMCs associate with arteries during development. A, Schematic diagram illustrating vSMC coverage of arteries and lack of coverage of veins. B, Schematic diagram of a zebrafish larva with the red box highlighting the area imaged in panel C. C, Confocal micrograph of the anterior trunk of a $5 \mathrm{dpf} T g(\operatorname{tag} \ln : e G F P), T g(k d r l: m C h e r r y-C A A X)$ double-transgenic zebrafish larva expressing eGFP in vSMCs (green) and mCherry-CAAX in the endothelium (magenta). vSMCs are associated with the dorsal aorta (DA) and not the cardinal vein (CV). D, Schematic diagram of a zebrafish larva with the red box highlighting the area imaged in panels $\mathrm{E}$ and F. E,F, Whole mount in situ hybridization of the mid-trunk of $48 \mathrm{hpf}$ zebrafish larvae probed for $c x c l 2 b$ ligand (E) or cxcr4a receptor (F). Expression of both genes is enriched in the dorsal aorta compared to the cardinal vein. G, Schematic representation of arterial-enriched expression of $c x c l 12 b$ and $c x c r 4 a$. Scale bars $=75 \mu \mathrm{m}$ (panel C).

Figure 2. Disrupting cxcl12b/cxcr4a signaling decreases vSMC association with arteries. A,B, Confocal images (left) and schematic representations (right) of the dorsal aorta (DA) in the anterior trunk of $5 \mathrm{dpf} T g($ tagln:eGFP), $T g(k d r l: m C h e r r y-C A A X)$ double-transgenic sibling (A) or cxcl12b424/424 mutant (B) zebrafish expressing eGFP in vSMCs (green) and mCherry-CAAX in the endothelium (magenta). C, D, Quantification of the number of associated vSMC (C) and width (D) of the dorsal aorta in $5 \mathrm{dpf} T g($ tagln:eGFP), $T g(k d r l: m C h e r r y-C A A X)$ double-transgenic sibling (black columns) or cxcl12b $424 / \Delta 24$ mutant (green columns) larvae. Values are expressed as a percentage of control siblings and averaged from three individual experiments. E,F, Confocal images (left) and schematic representations (right) of the dorsal aorta (DA) in the anterior trunk of 5 dpf $T g($ tagln:eGFP), Tg(kdrl:mCherry-CAAX) double-transgenic siblings (E) or cxcr4 $47 / \Delta 7$ mutant (F) zebrafish expressing eGFP in vSMCs (green) and mCherry-CAAX in the endothelium (magenta). G,H, Quantification of the number of associated vSMC (G) and width (H) of the dorsal aorta in $5 \mathrm{dpf} T g($ tagln:eGFP), $T g(k d r l: m C h e r r y-C A A X)$ double-transgenic sibling (black columns) or cxcr4 $47 / \Delta 7$ mutant (purple columns) larvae. Values are expressed as a percentage of control siblings and averaged from three individual experiments. I,J, Confocal images of immunohistochemically stained transverse sections through the dorsal aorta of E12.5 Cxcr4+/heterozygous sibling (I) and Cxcr4-/- mutant (J) mice, probed for platelet endothelial cell adhesion molecule-1 (PECAM) for endothelium (blue) and alpha smooth muscle actin (SMA) for vascular smooth muscle (vSMC, red). White brackets note the thickness of the vSMC layer surrounding 
the DA. K,L, Quantification of vSMC wall thickness (K) and lumenal area (L) of the dorsal aorta of E12.5 Cxcr4+/- heterozygous sibling (black columns) and Cxcr4-/- mutant (purple columns) mice, measured from immunohistochemically stained sections as in panels I and J. Values are expressed as a percentage of heterozygous siblings and averaged from three individual experiments. Scale bars $=75 \mu \mathrm{m}$ (panels A,B,E,F), $100 \mu \mathrm{m}$ (panels I,J). Box plots are graphed showing the median versus the first and third quartiles of the data (the middle, top, and bottom lines of the box respectively). The whiskers demonstrate the spread of data within $1.5 x$ above and below the interquartile range. All data points are shown as individual dots, with outliers shown above or below the whiskers. P-values are indicated above statistically significant datasets.

Figure 3. $c x c l 12 b$ promotes vSMC association without serving as a direct chemoattractant. $\mathbf{A}, \mathbf{B}$, Schematic diagrams illustrating the experimental design for using the mrcla promoter to drive ectopic mosaic expression of $c x c l 12 b$ in veins. A, A Tol2(mrcla:cxcl12b-2a-mCherry) DNA construct co-translationally expressing $c x c l 12 b$ and mCherry under the control of the mrcla promoter is injected into $T g(\operatorname{tag} \ln : e G F P)$ transgenic zebrafish embryos at the 1 cell stage. $\mathbf{B}$, At 4 dpf tol2(mrcla:cxcl12b-2a-mCherry)-injected zebrafish larvae are analyzed for vSMC (eGFP) association at sites of mCherry (i.e. $\mathrm{cxcl12b}$ ) expression in the dorsal aorta and cardinal vein. C,D, Representative confocal images of the mid-trunk of $4 \mathrm{dpf} T g(\operatorname{tag} \ln : e G F P$ ) transgenic larvae injected with either control Tol2(mrcla) "empty vector" (C) or Tol2(mrcla:cxcl12b-2a-mCherry) (D). eGFP-expressing vSMCs are shown in green, cxcl12b-2a-mCherry expression in dorsal aorta (DA) or cardinal vein (CV) endothelium is shown in magenta. E, Quantification of eGFP-positive vSMC associated with the dorsal aorta (DA) or cardinal vein (CV) in $4 \mathrm{dpf} T$ g (tagln:eGFP) transgenic zebrafish injected with either control Tol2(mrcla) "empty vector" (black columns) or Tol2(mrcla:cxcl12b-2a-mCherry) (green columns), showing strongly increased association of vSMCs with the cardinal vein. F, Schematic diagrams showing potential models for direct (left) versus indirect (right) mechanisms for promoting arterial recruitment of vSMC via CXCL12. G, Schematic diagram illustrating the 3D pulmonary artery smooth muscle cell (PASMC) motility assay. CXCL12, PDGFB, or nothing (control) is placed within the collagen gel to determine if PASMCs migrate towards these potential chemoattractants. H, Representative lateral images of 3D collagen gels showing PASMCs within the collagen matrix for each gel condition. I, Quantification of the relative number of PASMCs invading the collagen gel. The control is set to $100 \%$ and the CXCL12 and PDGFB conditions normalized to this level of invasion. Scale bars = 
$75 \mu \mathrm{m}$ (panels C,D), $200 \mu \mathrm{m}$ (panel H). Box plots are graphed showing the median versus the first and third quartiles of the data (the middle, top, and bottom lines of the box respectively). The whiskers demonstrate the spread of data within 1.5x above and below the interquartile range. All data points are shown as individual dots, with outliers shown above or below the whiskers. Pvalues are indicated above statistically significant datasets.

\section{Figure 4. $p d g f b$ mediated signaling regulates vSMC association with arteries.}

A, Schematic representation of the area imaged for in situ hybridization analysis, demonstrating the location of the dorsal aorta (DA) and cardinal vein (CV) within these images. B,C, Whole mount in situ hybridization (WISH) images of $p d g f b$ transcript (purple) in $1.5 \mathrm{dpf}$ (B) and $3 \mathrm{dpf}$ (C) zebrafish. Red dashed lines outline the dorsal aorta, blue dashed lines outline the cardinal vein. D, Quantification of the number of vSMC associated with the dorsal aorta in 5 dpf Tg(tagln:eGFP), Tg(kdrl:mCherry-CAAX) double-transgenic zebrafish carrying different combinations of heterozygous or homozygous $p d g f b a$ and $p d g f b b$ mutants. Values are averaged from three individual experiments and expressed as a percentage of the $p d g f b a+/-, p d g f b b_{+/+}$ control. E,F, Confocal images (left) and schematic representations (right) of the dorsal aorta (DA) in the anterior trunk of $5 \mathrm{dpf} T g(\operatorname{tag} \ln : e G F P), T g(k d r l: m C h e r r y-C A A X)$ double-transgenic

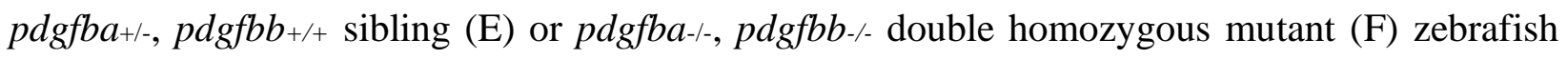
expressing eGFP in vSMCs (green) and mCherry-CAAX in the endothelium (magenta). G, Schematic diagram illustrating the proposed model for endothelial-autonomous chemokine signaling driving increased endothelial PDGFB ligand production, and thereby indirectly promoting vSMC acquisition by arteries. Scale bars $=75 \mu \mathrm{m}$ (panels E,F). Box plots are graphed showing the median versus the first and third quartiles of the data (the middle, top, and bottom lines of the box respectively). The whiskers demonstrate the spread of data within $1.5 x$ above and below the interquartile range. All data points are shown as individual dots, with outliers shown above or below the whiskers. P-values are indicated above statistically significant datasets.

Figure 5. Chemokine signaling regulates PDGFB transcript and protein levels across species. A,B, PDGFB transcript (A) and protein (B) in HUVEC cells cultured in vitro in a confluent cell monolayer for up to 8 hours with ("+CXCL12") or without ("CTRL") added recombinant CXCL12. Relative PDGFB transcript levels (A) and protein levels (B) were measured by qPCR and Western blot, respectively, showing an upregulation of both PDGFB transcript and PDGFB 
protein levels in response to stimulation by CXCL12. C-E, PDGFB transcript (C) and protein levels $(\mathrm{D}, \mathrm{E})$ in HUVEC cells cultured in vitro in a confluent cell monolayer and treated with either control, CXCR4, or CXCL12 siRNAs. Relative PDGFB transcript (C) and protein (E,F) levels were measured by qPCR and Western blot, respectively, showing suppression of both PDGFB transcript and protein in response to either CXCR4 or CXCL12 knockdown. Values in A, C, and E are averaged from three individual experiments and expressed as a percentage of control. Error bars \pm s.d. (A,C). F, Confocal images of immunohistochemically stained transverse sections through the dorsal aorta of E12.5 Cxcr4+/- heterozygous sibling (F) and Cxcr4-/- mutant (G) mice, probed for platelet derived growth factor B (PDGFB; green) and for smooth muscle 22 alpha (SM22, aka transgelin) for vascular smooth muscle cells (vSMC, red). G, Quantification of relative PDGFB protein expression in Cxcr4+/- heterozygous embryos versus Cxcr4-/- homozygous mutant embryos. Values are expressed as a percentage of heterozygous control and averaged from five individual mice per condition. H, Schematic diagram of a zebrafish larva with the red box highlighting the area imaged in panels I and J. I,J, Whole mount in situ hybridization of the midtrunk of 2.5 dpf zebrafish injected with control (I) or cxcl12b (J) RNA, showing upregulation of $p d g f b$ transcript in response to exogenous $c x c l 12 b$. Red and blue brackets in panel $\mathrm{J}$ indicate the dorsal aorta and cardinal vein, respectively. $\mathbf{K}$, Western blot of whole embryo protein lysate from $2.5 \mathrm{dpf}$ zebrafish injected with either control (left) or $c x c l 12 b$ (right) RNA, probed for pdgfb (top) or alpha tubulin (bottom), showing upregulation of $p d g f b$ protein levels in response to exogenous cxcl12b. Images are representative of data from three individual experiments. M, Schematic diagram illustrating the proposed model for endothelial-autonomous chemokine signaling driving increased endothelial PDGFB ligand production, thereby indirectly promoting vSMC acquisition by arteries. Putative upstream regulators of CXCL12 and CXCR4 are noted in red. Scale bars = $50 \mu \mathrm{m}$ (panel F). Box plots are graphed showing the median versus the first and third quartiles of the data (the middle, top, and bottom lines of the box respectively). The whiskers demonstrate the spread of data within $1.5 x$ above and below the interquartile range. All data points are shown as individual dots, with outliers shown above or below the whiskers. P-values are indicated above statistically significant datasets.

Figure 6. KLF2 is a negative regulator of chemokine signaling and PDGFB during early development. A) Top: Schematic diagram of a zebrafish larva with the red box highlighting the area imaged below. Bottom: Representative whole mount in situ hybridization (WISH) image of 
a 60 hpf zebrafish probed for klf2a. Red arrows indicate the dorsal aorta; blue arrows indicate the cardinal vein. B,C, Fluorescent images were taken of transverse sections through E9.5 mice: IHC for GFP was done to amplify signal from a Klf2-GFP knockin allele where the GFP is fused to the N-terminus of Klf2 (Klf2; green) and for platelet endothelial cell adhesion molecule-1 (PECAM) to mark the endothelium (red). Nuclei are labeled with DAPI (blue). Arrows highlight Klf2 positive endothelial nuclei in the cardinal vein (B) and vitelline vein (C) (Goddard et al., 2017; Weinreich et al., 2009). D,E, Quantitative qPCR measurement of CXCR4 (D) and PDGFB (E) transcript levels in HUVEC cells cultured in vitro as a confluent cell monolayer and treated with either control (black columns) or KLF2 (blue columns) siRNA. Values are expressed as a percentage of control. Error bars \pm s.d. F-H, Representative Western blot images of CXCR4 and PDGFB protein levels $(\mathrm{F})$, and quantification of relative CXCR4 $(\mathrm{G})$ and PDGFB protein levels (H) from HUVEC cells cultured in vitro in a confluent cell monolayer and treated with either control or KLF2 siRNA. Values in G,H are averaged from three individual experiments and expressed as a percentage of control. I,J, Quantification of vSMC number associated with the dorsal aorta (I) or cardinal vein (J) in the mid-trunk of $5 \mathrm{dpf}$ wild type sibling (black columns) or klf $2 a \Delta 8 / \Delta 8$ mutant (blue columns) animals. Values are averaged from three individual experiments and expressed as a percentage of wild type siblings. K, Quantification of dorsal aorta (DA, left columns) and cardinal vein ( $\mathrm{CV}$, right columns) width in the mid-trunk of $5 \mathrm{dpf}$ wild type sibling (black columns) or klf2a $\Delta 8 / \Delta 8$ mutant (blue columns) animals. L,M, Confocal images of the anterior trunk of 5 dpf $T g$ (tagln:eGFP), $T g(k d r l: m C h e r r y-C A A X)$ sibling (L) and klf2a $a / \Delta 8$ mutant (M) zebrafish embryos with eGFP positive vSMCs (green) and mCherry-CAAX positive endothelial cells (magenta). Arrows point to vSMCs associated with the CV in klf2a $a 8 / \Delta 8$ mutants. Scale bars $=75 \mu \mathrm{m}$ (panels L,M). Box plots are graphed showing the median versus the first and third quartiles of the data (the middle, top, and bottom lines of the box respectively). The whiskers demonstrate the spread of data within $1.5 \mathrm{x}$ above and below the interquartile range. All data points are shown as individual dots, with outliers shown above or below the whiskers. P-values are indicated above statistically significant datasets.

Figure 7. A proposed model for the preferential recruitment of vascular smooth muscle cells

to arteries. A, During the early development of arterial and venous blood vessels the CXCL12 ligand, its receptor CXCR4, and the vascular smooth muscle cell (vSMC) chemoattractant PDGFB are all more highly expressed on arteries than on veins. In contrast, KLF2 is more highly expressed 
on primitive veins than arteries. B, A proposed molecular pathway for preferential recruitment of vSMC to arteries. In arteries, autocrine activation of endothelial CXCR4 by its CXCL12 ligand results in increased production of PDGFB by arterial endothelium, promoting vSMC recruitment to arteries. Expression of KLF2 in primitive veins suppresses expression of CXCL12 and CXCR4, preventing up-regulation of PDGFB and limiting vSMC recruitment to veins. The cues directing preferential expression of KLF2 in veins remain unclear, but may involve different types of flow (ie, pulsatile vs. laminar).

Supplemental Figure 1. CRISPR/Cas9 mutant alleles. Gene structure and sequence of mutants generated for this study using CRISPR/Cas9 mutagenesis. A, cxcl12b 424 mutant. A 24 base pair insertion was introduced that destroys the splice donor site between exons 1 and 2 and inserts an in frame early stop codon. B, cxcr4a7 mutant. A 7 base pair deletion in exon 2 leads to a frame shift at amino acid 75 and early termination after amino acid 198. C, pdgfba $\Delta 6$ mutant. A 6 base pair deletion in exon 2 (chr22:29,325,360-29,325,754) leads to an in-frame deletion of two polar amino acids-a threonine and serine- leading to predicted alterations in protein folding. $\mathbf{D}, \boldsymbol{p d g} \boldsymbol{d} \boldsymbol{b} \boldsymbol{b}_{\boldsymbol{\Delta}}$ mutant. A 3 base pair deletion in exon 2 that leads to an in-frame deletion of a histidine leading to predicted alterations in protein folding. E, $k$ lf $2 \boldsymbol{a}_{\Delta 8}$ mutant. An 8 base pair insertion in exon 2 leads to a frame shift at amino acid 476 and early termination after amino acid 543.

\section{Supplemental Figure 2. siRNA knockdown in Human Umbilical Vein Endothelial Cells}

(HUVEC). We confirmed suppression of genes targeted by siRNAs in HUVECs for this study. Transcript levels were measured by performing qRT-PCR for each respective gene, comparing control siRNA treated to PDGFB (A), CXCL12 (B), CXCR4 (C), or KLF2 (D) siRNA treated cell samples. Data are representative of 3 experimental replicates. Values are normalized to control siRNA levels. Error bars \pm s.d. 


\section{References:}

Abramsson, A., Kurup, S., Busse, M., Yamada, S., Lindblom, P., Schallmeiner, E., Stenzel, D., Sauvaget, D., Ledin, J., Ringvall, M., et al. (2007). Defective N-sulfation of heparan sulfate proteoglycans limits PDGF-BB binding and pericyte recruitment in vascular development. Genes Dev 21, 316-331.

Adam, P.J., Regan, C.P., Hautmann, M.B., and Owens, G.K. (2000). Positive- and negative-acting Kruppel-like transcription factors bind a transforming growth factor beta control element required for expression of the smooth muscle cell differentiation marker SM22alpha in vivo. J Biol Chem $275,37798-37806$.

Ando, K., Fukuhara, S., Izumi, N., Nakajima, H., Fukui, H., Kelsh, R.N., and Mochizuki, N. (2016). Clarification of mural cell coverage of vascular endothelial cells by live imaging of zebrafish. Development 143, 1328-1339.

Arkenbout, E.K., Dekker, R.J., de Vries, C.J., Horrevoets, A.J., and Pannekoek, H. (2003). Focusing on transcription factor families in atherogenesis: the function of LKLF and TR3. Thromb Haemost 89, 522-529.

Atkins, G.B., and Jain, M.K. (2007). Role of Kruppel-like transcription factors in endothelial biology. Circ Res 100, 1686-1695.

Bergers, G., and Song, S. (2005). The role of pericytes in blood-vessel formation and maintenance. Neuro Oncol 7, 452-464.

Bhattacharya, R., Senbanerjee, S., Lin, Z., Mir, S., Hamik, A., Wang, P., Mukherjee, P., Mukhopadhyay, D., and Jain, M.K. (2005). Inhibition of vascular permeability factor/vascular endothelial growth factor-mediated angiogenesis by the Kruppel-like factor KLF2. J Biol Chem $280,28848-28851$.

Busillo, J.M., and Benovic, J.L. (2007). Regulation of CXCR4 signaling. Biochim Biophys Acta $1768,952-963$.

Bussmann, J., Wolfe, S.A., and Siekmann, A.F. (2011). Arterial-venous network formation during brain vascularization involves hemodynamic regulation of chemokine signaling. Development 138, 1717-1726.

Castro, M., Lavina, B., Ando, K., Alvarez-Aznar, A., Abu Taha, A., Brakebusch, C., Dejana, E., Betsholtz, C., and Gaengel, K. (2019). CDC42 Deletion Elicits Cerebral Vascular Malformations via Increased MEKK3-Dependent KLF4 Expression. Circ Res 124, 1240-1252.

Cha, Y.R., Fujita, M., Butler, M., Isogai, S., Kochhan, E., Siekmann, A.F., and Weinstein, B.M. (2012). Chemokine signaling directs trunk lymphatic network formation along the preexisting blood vasculature. Dev Cell 22, 824-836. 
Cha, Y.R., and Weinstein, B.M. (2012). Use of PCR template-derived probes prevents off-target whole mount in situ hybridization in transgenic zebrafish. Zebrafish 9, 85-89.

Chang, L., Noseda, M., Higginson, M., Ly, M., Patenaude, A., Fuller, M., Kyle, A.H., Minchinton, A.I., Puri, M.C., Dumont, D.J., et al. (2012). Differentiation of vascular smooth muscle cells from local precursors during embryonic and adult arteriogenesis requires Notch signaling. Proc Natl Acad Sci U S A 109, 6993-6998.

Chiplunkar, A.R., Curtis, B.C., Eades, G.L., Kane, M.S., Fox, S.J., Haar, J.L., and Lloyd, J.A. (2013). The Kruppel-like factor 2 and Kruppel-like factor 4 genes interact to maintain endothelial integrity in mouse embryonic vasculogenesis. BMC Dev Biol 13, 40.

Claxton, S., Kostourou, V., Jadeja, S., Chambon, P., Hodivala-Dilke, K., and Fruttiger, M. (2008). Efficient, inducible Cre-recombinase activation in vascular endothelium. Genesis 46, 74-80.

Corti, P., Young, S., Chen, C.Y., Patrick, M.J., Rochon, E.R., Pekkan, K., and Roman, B.L. (2011). Interaction between alk1 and blood flow in the development of arteriovenous malformations. Development 138, 1573-1582.

Dandre, F., and Owens, G.K. (2004). Platelet-derived growth factor-BB and Ets-1 transcription factor negatively regulate transcription of multiple smooth muscle cell differentiation marker genes. Am J Physiol Heart Circ Physiol 286, H2042-2051.

Davies, P.F., Civelek, M., Fang, Y., and Fleming, I. (2013). The atherosusceptible endothelium: endothelial phenotypes in complex haemodynamic shear stress regions in vivo. Cardiovasc Res 99, 315-327.

Dekker, R.J., Boon, R.A., Rondaij, M.G., Kragt, A., Volger, O.L., Elderkamp, Y.W., Meijers, J.C., Voorberg, J., Pannekoek, H., and Horrevoets, A.J. (2006). KLF2 provokes a gene expression pattern that establishes functional quiescent differentiation of the endothelium. Blood 107, 43544363.

Dekker, R.J., van Soest, S., Fontijn, R.D., Salamanca, S., de Groot, P.G., VanBavel, E., Pannekoek, H., and Horrevoets, A.J. (2002). Prolonged fluid shear stress induces a distinct set of endothelial cell genes, most specifically lung Kruppel-like factor (KLF2). Blood 100, 1689-1698.

Dekker, R.J., van Thienen, J.V., Rohlena, J., de Jager, S.C., Elderkamp, Y.W., Seppen, J., de Vries, C.J., Biessen, E.A., van Berkel, T.J., Pannekoek, H., et al. (2005). Endothelial KLF2 links local arterial shear stress levels to the expression of vascular tone-regulating genes. Am J Pathol 167, 609-618.

Doring, Y., Pawig, L., Weber, C., and Noels, H. (2014). The CXCL12/CXCR4 chemokine ligand/receptor axis in cardiovascular disease. Front Physiol 5, 212. 
Franzoni, M., O'Connor, D.T., Marcar, L., Power, D., Moloney, M.A., Kavanagh, E.G., Leask, R.L., Nolan, J., Kiely, P.A., and Walsh, M.T. (2019). The Presence of a High Peak Feature Within Low-Average Shear Stimuli Induces Quiescence in Venous Endothelial Cells. Ann Biomed Eng.

Fujita, M., Cha, Y.R., Pham, V.N., Sakurai, A., Roman, B.L., Gutkind, J.S., and Weinstein, B.M. (2011). Assembly and patterning of the vascular network of the vertebrate hindbrain. Development $138,1705-1715$.

Gimbrone, M.A., Jr., and Garcia-Cardena, G. (2013). Vascular endothelium, hemodynamics, and the pathobiology of atherosclerosis. Cardiovasc Pathol 22, 9-15.

Goddard, L.M., Duchemin, A.L., Ramalingan, H., Wu, B., Chen, M., Bamezai, S., Yang, J., Li, L., Morley, M.P., Wang, T., et al. (2017). Hemodynamic Forces Sculpt Developing Heart Valves through a KLF2-WNT9B Paracrine Signaling Axis. Dev Cell 43, 274-289 e275.

Gupta, S.K., Lysko, P.G., Pillarisetti, K., Ohlstein, E., and Stadel, J.M. (1998). Chemokine receptors in human endothelial cells. Functional expression of CXCR4 and its transcriptional regulation by inflammatory cytokines. J Biol Chem 273, 4282-4287.

Hall-Glenn, F., De Young, R.A., Huang, B.L., van Handel, B., Hofmann, J.J., Chen, T.T., Choi, A., Ong, J.R., Benya, P.D., Mikkola, H., et al. (2012). CCN2/connective tissue growth factor is essential for pericyte adhesion and endothelial basement membrane formation during angiogenesis. PLoS One 7, e30562.

Hamdan, R., Zhou, Z., and Kleinerman, E.S. (2011). SDF-1alpha induces PDGF-B expression and the differentiation of bone marrow cells into pericytes. Mol Cancer Res 9, 1462-1470.

Hamdan, R., Zhou, Z., and Kleinerman, E.S. (2014). Blocking SDF-1alpha/CXCR4 downregulates PDGF-B and inhibits bone marrow-derived pericyte differentiation and tumor vascular expansion in Ewing tumors. Mol Cancer Ther 13, 483-491.

Harrison, M.R., Bussmann, J., Huang, Y., Zhao, L., Osorio, A., Burns, C.G., Burns, C.E., Sucov, H.M., Siekmann, A.F., and Lien, C.L. (2015). Chemokine-guided angiogenesis directs coronary vasculature formation in zebrafish. Dev Cell 33, 442-454.

Hedin, U., Roy, J., Tran, P.K., Lundmark, K., and Rahman, A. (1999). Control of smooth muscle cell proliferation--the role of the basement membrane. Thromb Haemost 82 Suppl 1, 23-26.

Heickendorff, L. (1989). The basement membrane of arterial smooth muscle cells. APMIS Suppl 9, 1-32.

Hellstrom, M., Kalen, M., Lindahl, P., Abramsson, A., and Betsholtz, C. (1999). Role of PDGF-B and PDGFR-beta in recruitment of vascular smooth muscle cells and pericytes during embryonic blood vessel formation in the mouse. Development 126, 3047-3055. 
Huddleson, J.P., Srinivasan, S., Ahmad, N., and Lingrel, J.B. (2004). Fluid shear stress induces endothelial KLF2 gene expression through a defined promoter region. Biol Chem 385, 723-729.

Jung, H.M., Castranova, D., Swift, M.R., Pham, V.N., Venero Galanternik, M., Isogai, S., Butler, M.G., Mulligan, T.S., and Weinstein, B.M. (2017). Development of the larval lymphatic system in zebrafish. Development 144, 2070-2081.

Kimmel, C.B., Ballard, W.W., Kimmel, S.R., Ullmann, B., and Schilling, T.F. (1995). Stages of embryonic development of the zebrafish. Dev Dyn 203, 253-310.

Koh, W., Stratman, A.N., Sacharidou, A., and Davis, G.E. (2008). In vitro three dimensional collagen matrix models of endothelial lumen formation during vasculogenesis and angiogenesis. Methods Enzymol 443, 83-101.

Komaravolu, R.K., Adam, C., Moonen, J.R., Harmsen, M.C., Goebeler, M., and Schmidt, M. (2015). Erk5 inhibits endothelial migration via KLF2-dependent down-regulation of PAK1. Cardiovasc Res 105, 86-95.

Kuo, C.T., Veselits, M.L., Barton, K.P., Lu, M.M., Clendenin, C., and Leiden, J.M. (1997). The LKLF transcription factor is required for normal tunica media formation and blood vessel stabilization during murine embryogenesis. Genes Dev 11, 2996-3006.

Le, V.P., Knutsen, R.H., Mecham, R.P., and Wagenseil, J.E. (2011). Decreased aortic diameter and compliance precedes blood pressure increases in postnatal development of elastin-insufficient mice. Am J Physiol Heart Circ Physiol 301, H221-229.

Lee, J.S., Yu, Q., Shin, J.T., Sebzda, E., Bertozzi, C., Chen, M., Mericko, P., Stadtfeld, M., Zhou, D., Cheng, L., et al. (2006). Klf2 is an essential regulator of vascular hemodynamic forces in vivo. Dev Cell 11, 845-857.

Lee, V.S., Halabi, C.M., Broekelmann, T.J., Trackman, P.C., Stitziel, N.O., and Mecham, R.P. (2019). Intracellular retention of mutant lysyl oxidase leads to aortic dilation in response to increased hemodynamic stress. JCI Insight 5.

Leveen, P., Pekny, M., Gebre-Medhin, S., Swolin, B., Larsson, E., and Betsholtz, C. (1994). Mice deficient for PDGF B show renal, cardiovascular, and hematological abnormalities. Genes Dev 8 , $1875-1887$.

Li, J., Zhao, Y., Coleman, P., Chen, J., Ting, K.K., Choi, J.P., Zheng, X., Vadas, M.A., and Gamble, J.R. (2019). Low fluid shear stress conditions contribute to activation of cerebral cavernous malformation signalling pathways. Biochim Biophys Acta Mol Basis Dis 1865, 165519.

Li, W., Kohara, H., Uchida, Y., James, J.M., Soneji, K., Cronshaw, D.G., Zou, Y.R., Nagasawa, T., and Mukouyama, Y.S. (2013). Peripheral nerve-derived CXCL12 and VEGF-A regulate the patterning of arterial vessel branching in developing limb skin. Dev Cell 24, 359-371. 
Lilly, B. (2014). We have contact: endothelial cell-smooth muscle cell interactions. Physiology (Bethesda) 29, 234-241.

Lin, C.H., and Lilly, B. (2014). Notch signaling governs phenotypic modulation of smooth muscle cells. Vascul Pharmacol 63, 88-96.

Lin, Z., Natesan, V., Shi, H., Dong, F., Kawanami, D., Mahabeleshwar, G.H., Atkins, G.B., Nayak, L., Cui, Y., Finigan, J.H., et al. (2010). Kruppel-like factor 2 regulates endothelial barrier function. Arterioscler Thromb Vasc Biol 30, 1952-1959.

Lindahl, P., Johansson, B.R., Leveen, P., and Betsholtz, C. (1997). Pericyte loss and microaneurysm formation in PDGF-B-deficient mice. Science 277, 242-245.

Lindblom, P., Gerhardt, H., Liebner, S., Abramsson, A., Enge, M., Hellstrom, M., Backstrom, G., Fredriksson, S., Landegren, U., Nystrom, H.C., et al. (2003). Endothelial PDGF-B retention is required for proper investment of pericytes in the microvessel wall. Genes Dev 17, 1835-1840.

Liu, Y., Sinha, S., McDonald, O.G., Shang, Y., Hoofnagle, M.H., and Owens, G.K. (2005). Kruppel-like factor 4 abrogates myocardin-induced activation of smooth muscle gene expression. J Biol Chem 280, 9719-9727.

Mack, P.J., Zhang, Y., Chung, S., Vickerman, V., Kamm, R.D., and Garcia-Cardena, G. (2009). Biomechanical Regulation of Endothelium-dependent Events Critical for Adaptive Remodeling. J Biol Chem 284, 8412-8420.

Methe, H., Balcells, M., Alegret Mdel, C., Santacana, M., Molins, B., Hamik, A., Jain, M.K., and Edelman, E.R. (2007). Vascular bed origin dictates flow pattern regulation of endothelial adhesion molecule expression. Am J Physiol Heart Circ Physiol 292, H2167-2175.

Nayak, L., Lin, Z., and Jain, M.K. (2011). "Go with the flow": how Kruppel-like factor 2 regulates the vasoprotective effects of shear stress. Antioxid Redox Signal 15, 1449-1461.

Noels, H., Zhou, B., Tilstam, P.V., Theelen, W., Li, X., Pawig, L., Schmitz, C., Akhtar, S., Simsekyilmaz, S., Shagdarsuren, E., et al. (2014). Deficiency of endothelial CXCR4 reduces reendothelialization and enhances neointimal hyperplasia after vascular injury in atherosclerosisprone mice. Arterioscler Thromb Vasc Biol 34, 1209-1220.

Novodvorsky, P., and Chico, T.J. (2014). The role of the transcription factor KLF2 in vascular development and disease. Prog Mol Biol Transl Sci 124, 155-188.

Osei-Owusu, P., Knutsen, R.H., Kozel, B.A., Dietrich, H.H., Blumer, K.J., and Mecham, R.P. (2014). Altered reactivity of resistance vasculature contributes to hypertension in elastin insufficiency. Am J Physiol Heart Circ Physiol 306, H654-666.

Owens, G.K., Kumar, M.S., and Wamhoff, B.R. (2004). Molecular regulation of vascular smooth muscle cell differentiation in development and disease. Physiol Rev 84, 767-801. 
Parmar, K.M., Larman, H.B., Dai, G., Zhang, Y., Wang, E.T., Moorthy, S.N., Kratz, J.R., Lin, Z., Jain, M.K., Gimbrone, M.A., Jr., et al. (2006). Integration of flow-dependent endothelial phenotypes by Kruppel-like factor 2. J Clin Invest 116, 49-58.

Payne, S., De Val, S., and Neal, A. (2018). Endothelial-Specific Cre Mouse Models. Arterioscler Thromb Vasc Biol 38, 2550-2561.

Renz, M., Otten, C., Faurobert, E., Rudolph, F., Zhu, Y., Boulday, G., Duchene, J., Mickoleit, M., Dietrich, A.C., Ramspacher, C., et al. (2015). Regulation of betal integrin-Klf2-mediated angiogenesis by CCM proteins. Dev Cell 32, 181-190.

Roostalu, U., and Wong, J.K. (2018). Arterial smooth muscle dynamics in development and repair. Dev Biol 435, 109-121.

Sangwung, P., Zhou, G., Nayak, L., Chan, E.R., Kumar, S., Kang, D.W., Zhang, R., Liao, X., Lu, Y., Sugi, K., et al. (2017). KLF2 and KLF4 control endothelial identity and vascular integrity. JCI Insight 2, e91700.

Santoro, M.M., Pesce, G., and Stainier, D.Y. (2009). Characterization of vascular mural cells during zebrafish development. Mech Dev 126, 638-649.

Scheppke, L., Murphy, E.A., Zarpellon, A., Hofmann, J.J., Merkulova, A., Shields, D.J., Weis, S.M., Byzova, T.V., Ruggeri, Z.M., Iruela-Arispe, M.L., et al. (2012). Notch promotes vascular maturation by inducing integrin-mediated smooth muscle cell adhesion to the endothelial basement membrane. Blood 119, 2149-2158.

Sebzda, E., Zou, Z., Lee, J.S., Wang, T., and Kahn, M.L. (2008). Transcription factor KLF2 regulates the migration of naive $\mathrm{T}$ cells by restricting chemokine receptor expression patterns. Nat Immunol 9, 292-300.

Sen-Banerjee, S., Mir, S., Lin, Z., Hamik, A., Atkins, G.B., Das, H., Banerjee, P., Kumar, A., and Jain, M.K. (2005). Kruppel-like factor 2 as a novel mediator of statin effects in endothelial cells. Circulation 112, 720-726.

Shi, X., DiRenzo, D., Guo, L.W., Franco, S.R., Wang, B., Seedial, S., and Kent, K.C. (2014). TGFbeta/Smad3 stimulates stem cell/developmental gene expression and vascular smooth muscle cell de-differentiation. PLoS One 9, e93995.

Siekmann, A.F., Standley, C., Fogarty, K.E., Wolfe, S.A., and Lawson, N.D. (2009). Chemokine signaling guides regional patterning of the first embryonic artery. Genes Dev 23, 2272-2277.

Stanczuk, L., Martinez-Corral, I., Ulvmar, M.H., Zhang, Y., Lavina, B., Fruttiger, M., Adams, R.H., Saur, D., Betsholtz, C., Ortega, S., et al. (2015). cKit Lineage Hemogenic EndotheliumDerived Cells Contribute to Mesenteric Lymphatic Vessels. Cell Rep 10, 1708-1721. 
Stratman, A.N., and Davis, G.E. (2012). Endothelial cell-pericyte interactions stimulate basement membrane matrix assembly: influence on vascular tube remodeling, maturation, and stabilization. Microsc Microanal 18, 68-80.

Stratman, A.N., Davis, M.J., and Davis, G.E. (2011). VEGF and FGF prime vascular tube morphogenesis and sprouting directed by hematopoietic stem cell cytokines. Blood 117, 37093719.

Stratman, A.N., Malotte, K.M., Mahan, R.D., Davis, M.J., and Davis, G.E. (2009). Pericyte recruitment during vasculogenic tube assembly stimulates endothelial basement membrane matrix formation. Blood 114, 5091-5101.

Stratman, A.N., Pezoa, S.A., Farrelly, O.M., Castranova, D., Dye, L.E., 3rd, Butler, M.G., Sidik, H., Talbot, W.S., and Weinstein, B.M. (2017). Interactions between mural cells and endothelial cells stabilize the developing zebrafish dorsal aorta. Development 144, 115-127.

Stratman, A.N., Schwindt, A.E., Malotte, K.M., and Davis, G.E. (2010). Endothelial-derived PDGF-BB and HB-EGF coordinately regulate pericyte recruitment during vasculogenic tube assembly and stabilization. Blood 116, 4720-4730.

van Thienen, J.V., Fledderus, J.O., Dekker, R.J., Rohlena, J., van Ijzendoorn, G.A., Kootstra, N.A., Pannekoek, H., and Horrevoets, A.J. (2006). Shear stress sustains atheroprotective endothelial KLF2 expression more potently than statins through mRNA stabilization. Cardiovasc Res 72, 231240.

Weinreich, M.A., Takada, K., Skon, C., Reiner, S.L., Jameson, S.C., and Hogquist, K.A. (2009). KLF2 transcription-factor deficiency in $\mathrm{T}$ cells results in unrestrained cytokine production and upregulation of bystander chemokine receptors. Immunity 31, 122-130.

Westerfield, M. (1995). The Zebrafish Book (Eugene, OR: University of Oregon Press).

Whitesell, T.R., Kennedy, R.M., Carter, A.D., Rollins, E.L., Georgijevic, S., Santoro, M.M., and Childs, S.J. (2014). An alpha-smooth muscle actin (acta2/alphasma) zebrafish transgenic line marking vascular mural cells and visceral smooth muscle cells. PLoS One 9, e90590.

Wiens, K.M., Lee, H.L., Shimada, H., Metcalf, A.E., Chao, M.Y., and Lien, C.L. (2010). Plateletderived growth factor receptor beta is critical for zebrafish intersegmental vessel formation. PLoS One 5, e11324.

Wu, J., Bohanan, C.S., Neumann, J.C., and Lingrel, J.B. (2008). KLF2 transcription factor modulates blood vessel maturation through smooth muscle cell migration. J Biol Chem 283, 39423950.

Zhou, Z., Tang, A.T., Wong, W.Y., Bamezai, S., Goddard, L.M., Shenkar, R., Zhou, S., Yang, J., Wright, A.C., Foley, M., et al. (2016). Cerebral cavernous malformations arise from endothelial gain of MEKK3-KLF2/4 signalling. Nature 532, 122-126. 


\section{Stratman, et al. Figure 1}

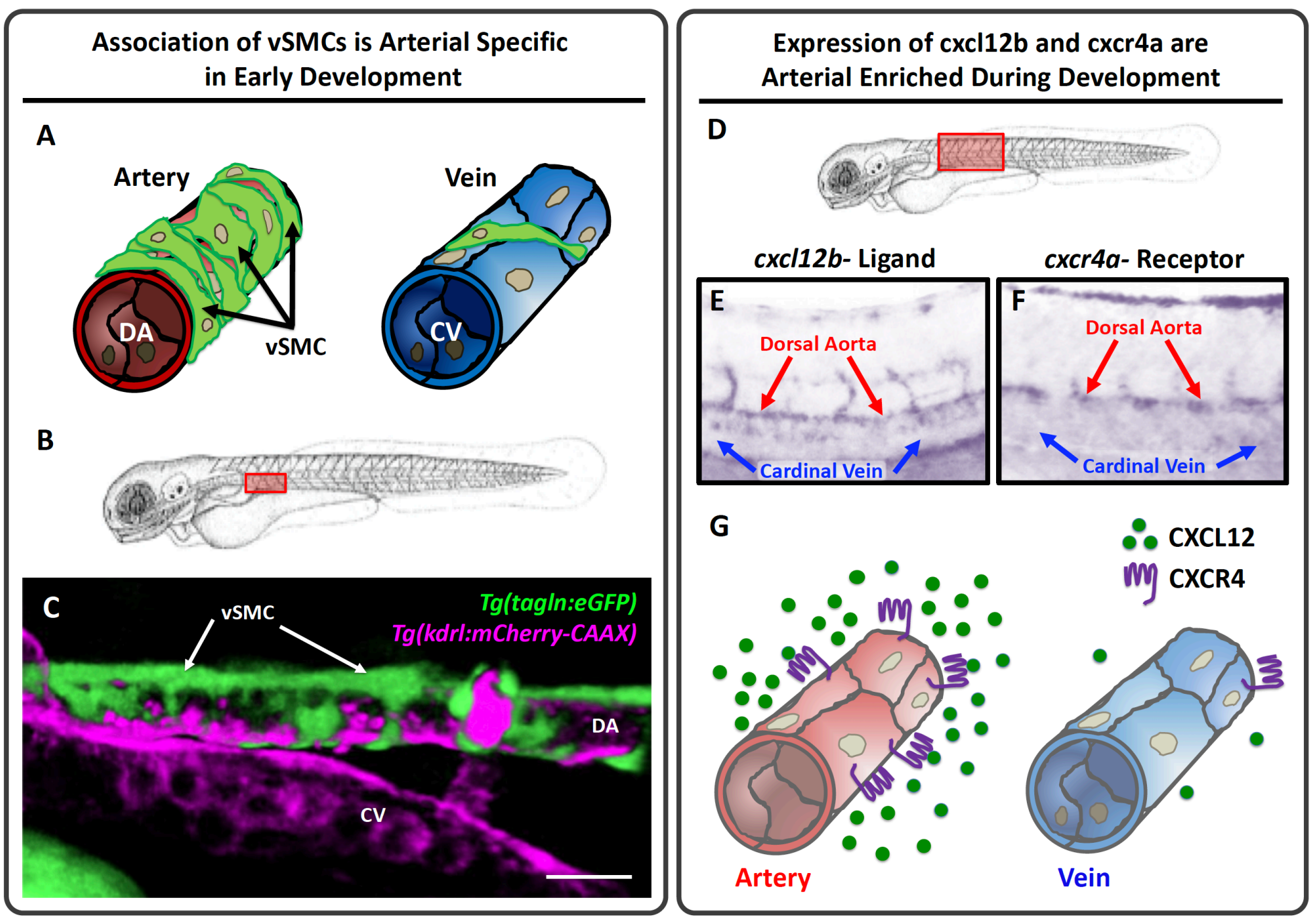


bioRxiv preprint doi: https://doi.org/10.1101/2019.12.27.889782; this version posted December 28, 2019. The copyright holder for this preprint (which was not certified by peer review) is the author/funder. All rights reserved. No reuse allowed without permission.

Stratman, et al. Figure 2
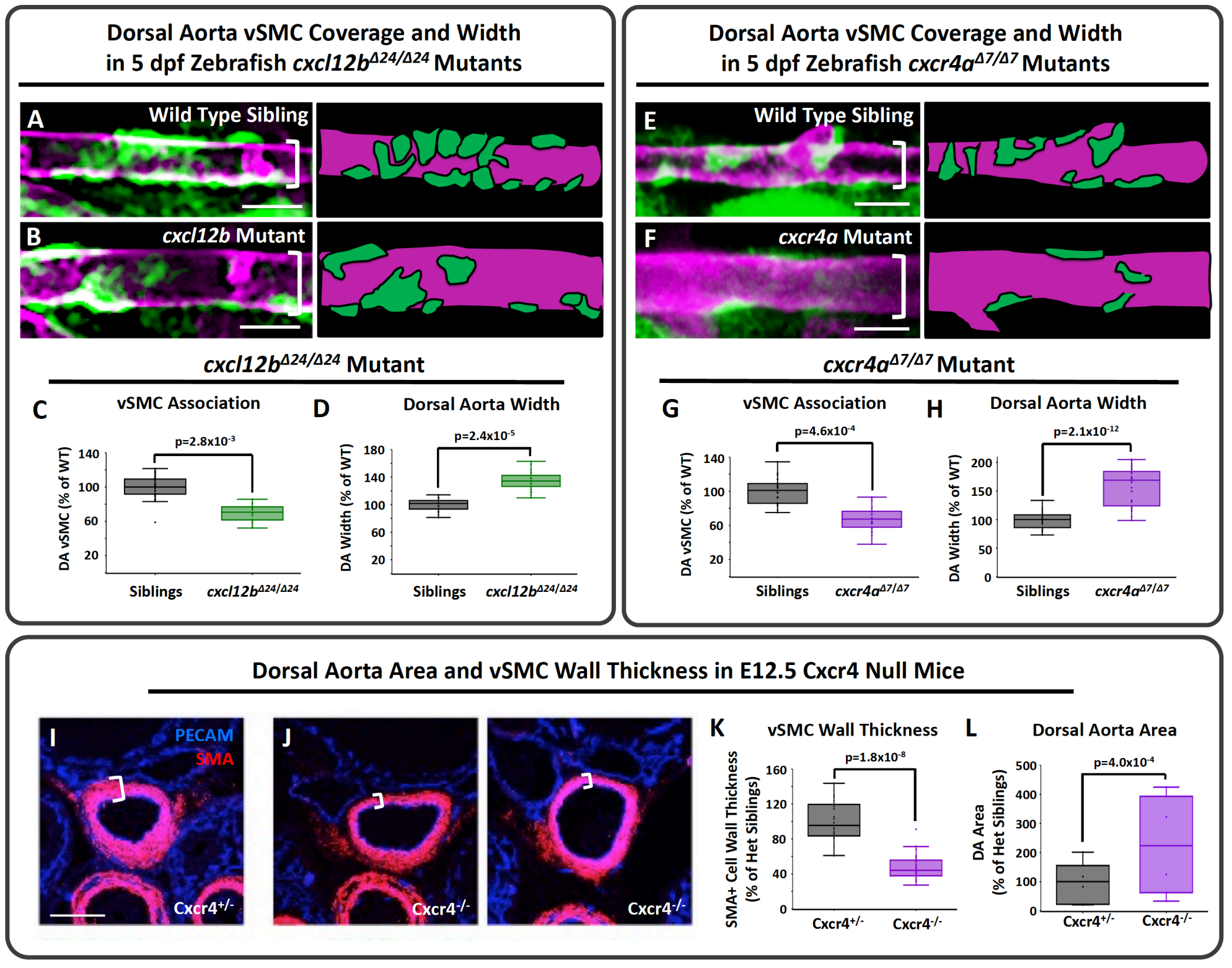


\section{Stratman, et al. Figure 3}

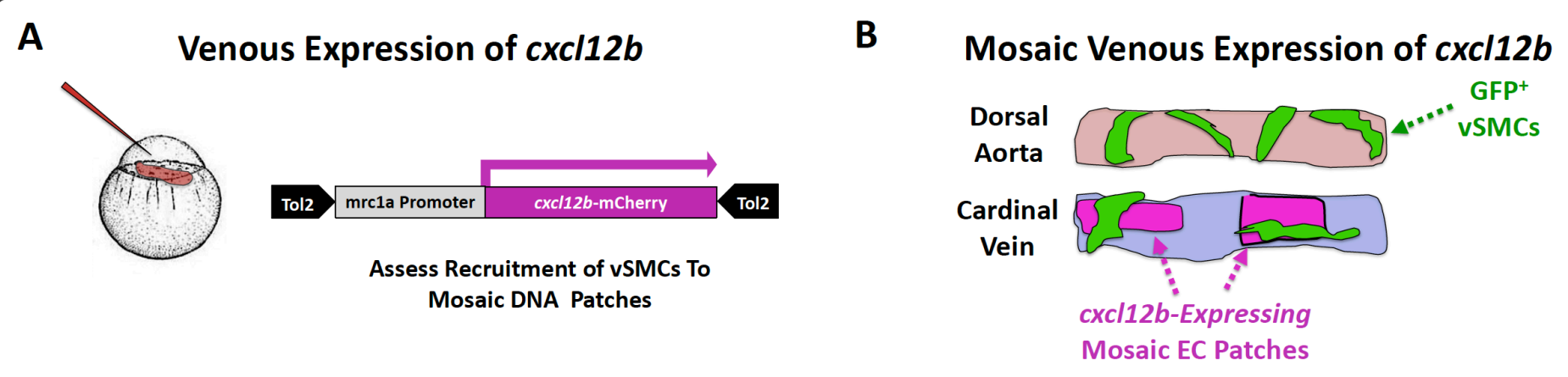

Analysis of vSMC Association to Mosaic $c x c / 12 b$ DNA Patches
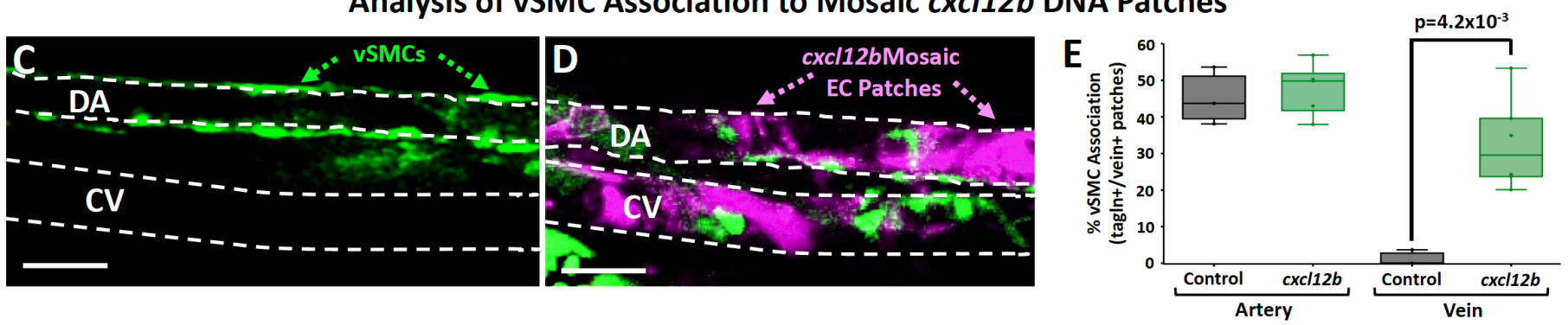

$\mathbf{F}$

Direct vs. Indirect Signaling to vSMC via CXCL12
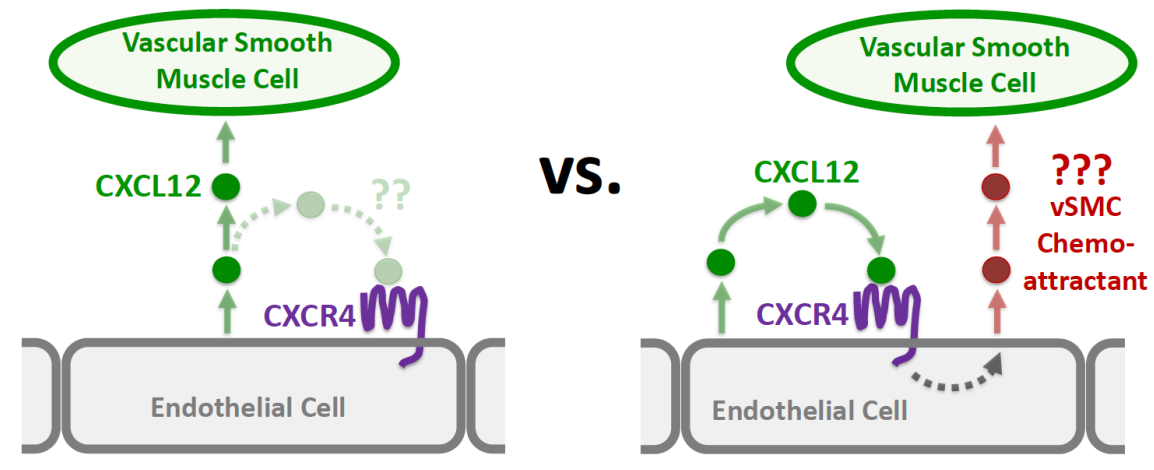

\section{G In Vitro 3-D Cell Motility Assay}

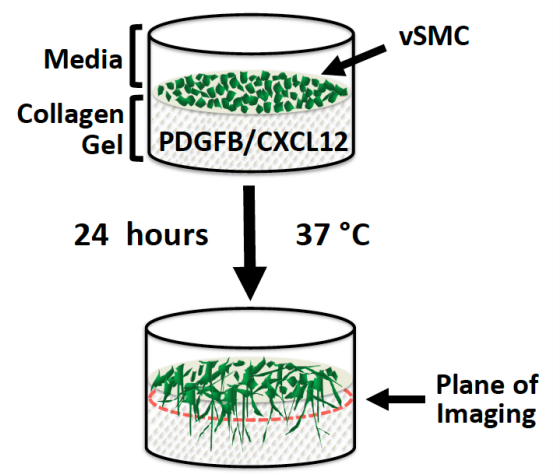

PDGFB is a vSMC Chemoattractant in 3D in vitro Cell Motility Assays

H

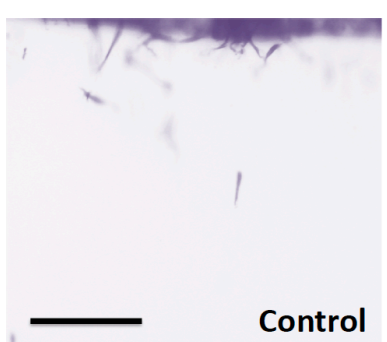

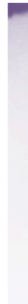

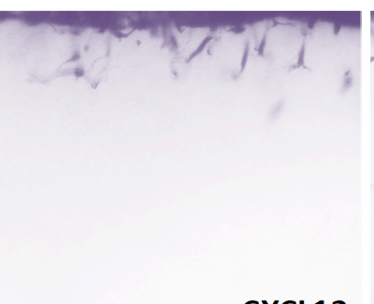

CXCL12

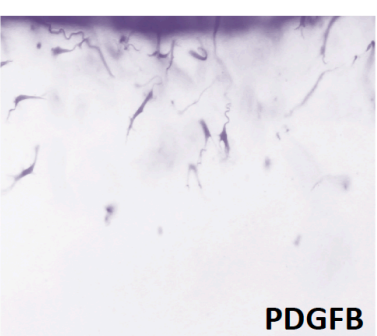

PDGFB

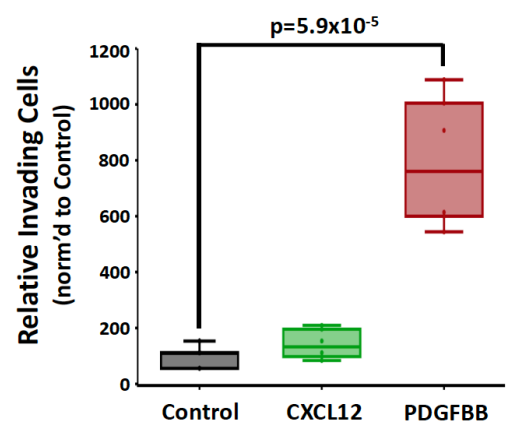




\section{Stratman, et al. Figure 4}
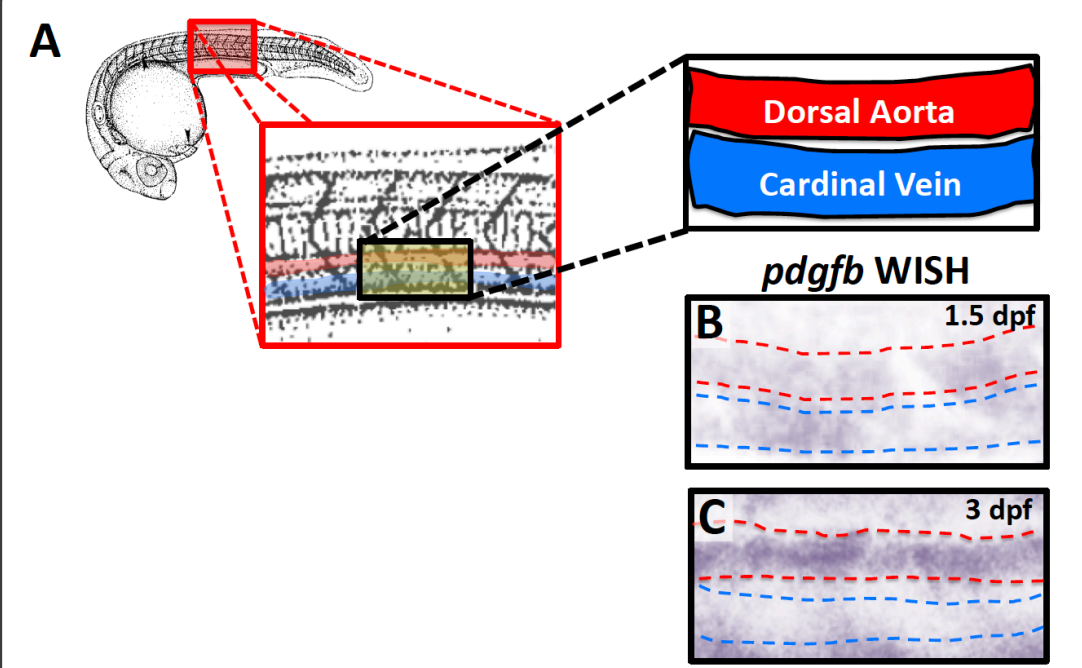

D
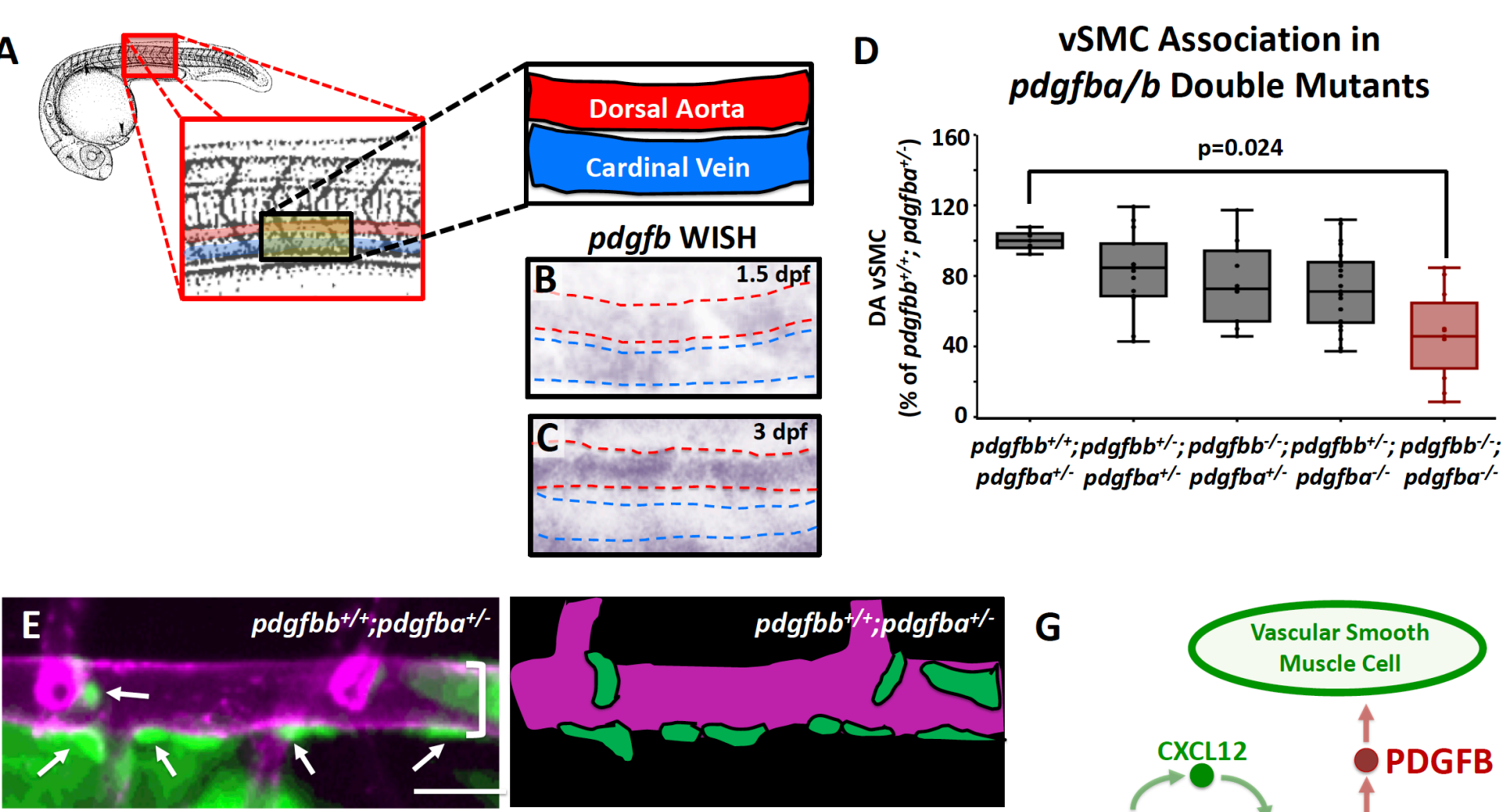

$\mathbf{G}$

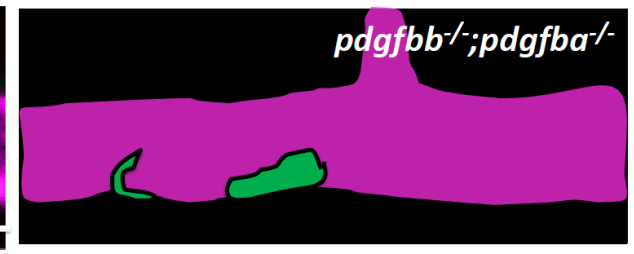

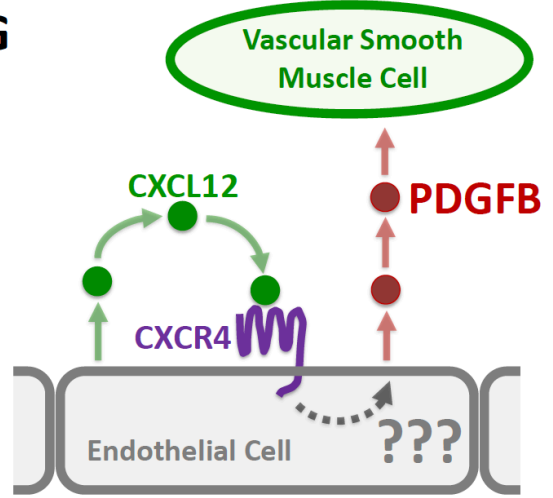




\section{Stratman, et al. Figure 5}
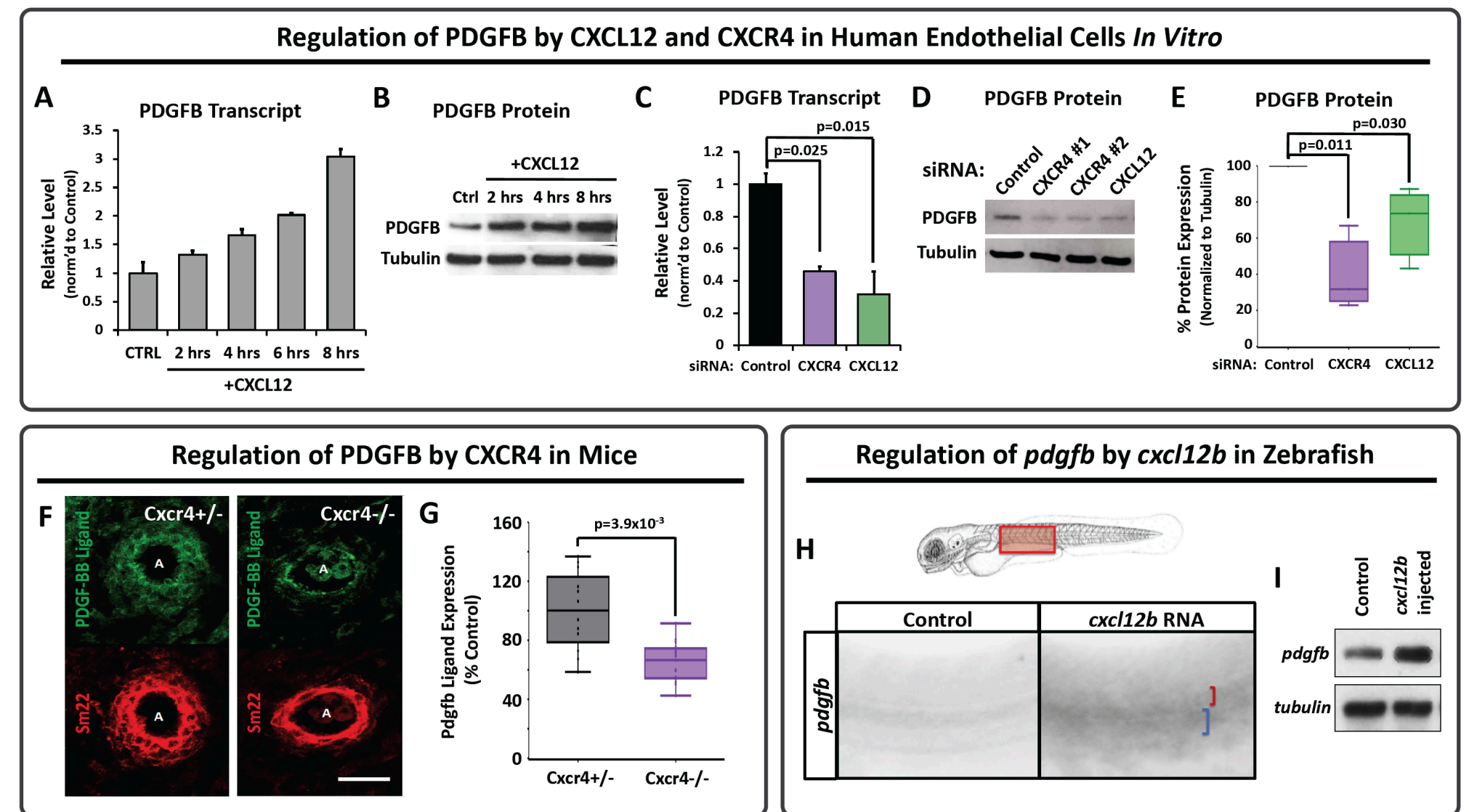

\section{Regulation of pdgfb by cxcl12b in Zebrafish}
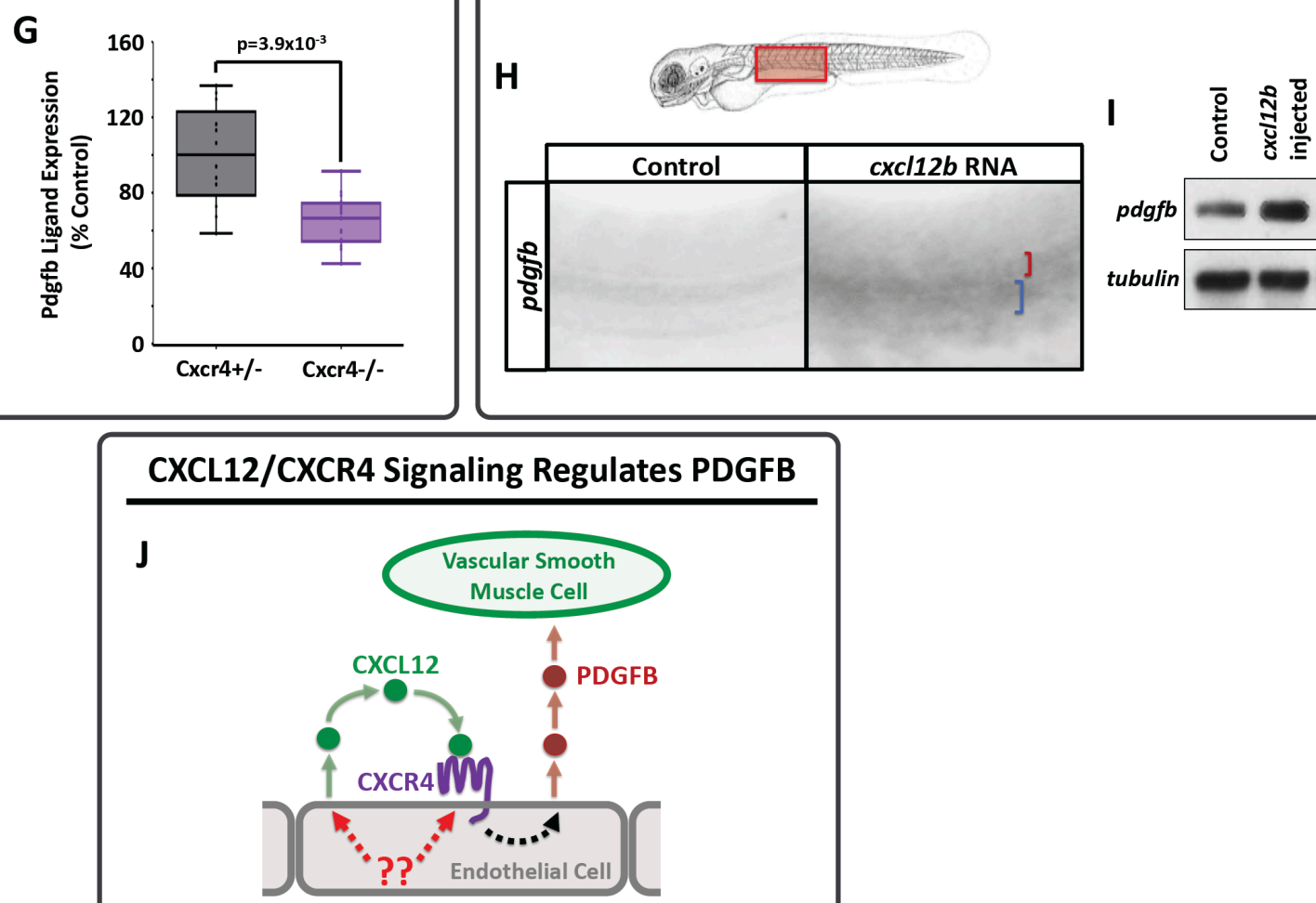


\section{Stratman, et al. Figure 6}

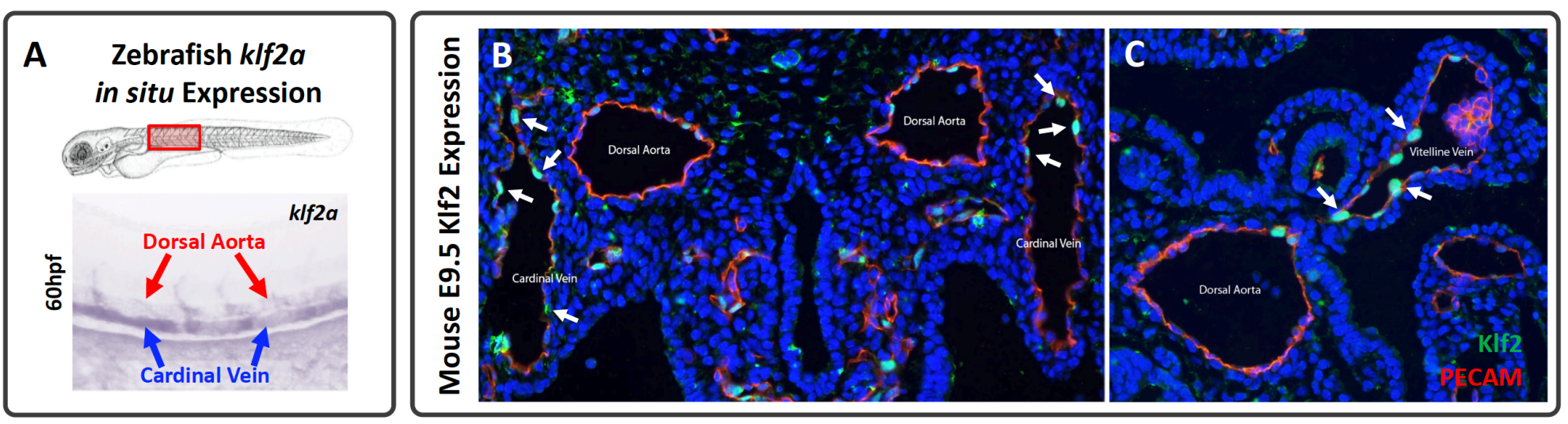

Transcript Levels in KLF2-Deficient Human EC
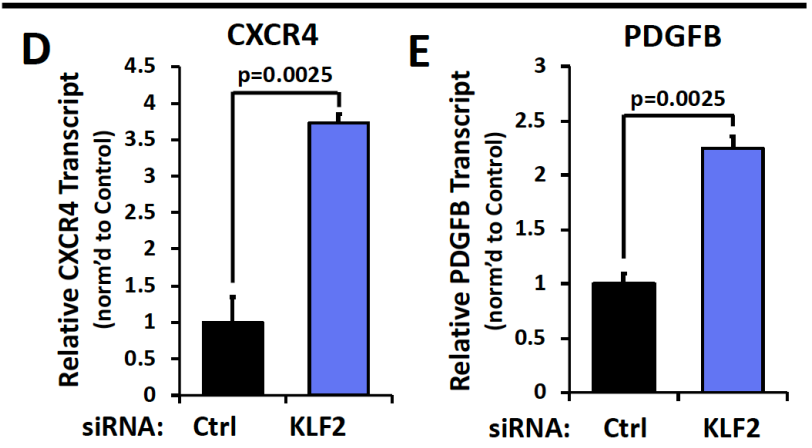

Protein Levels in KLF2-Deficient Human EC

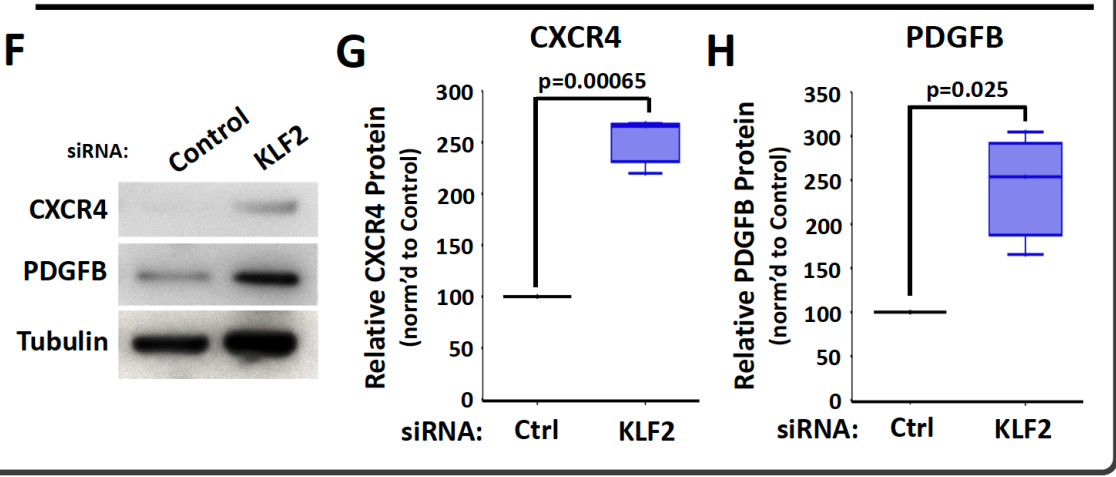

\section{$k I f 2 a^{\Delta 8 / \Delta 8}$ Zebrafish Mutant Analysis}

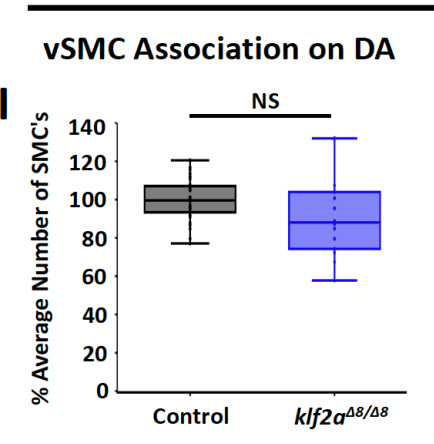

VSMC Association on CV

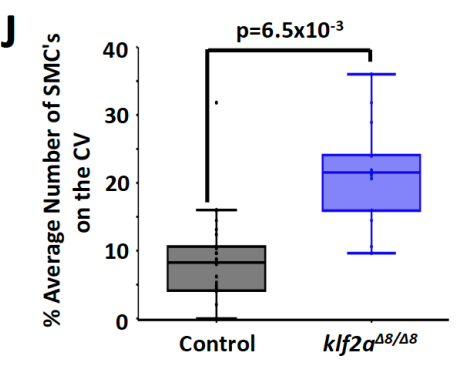

Vessel Width Analysis

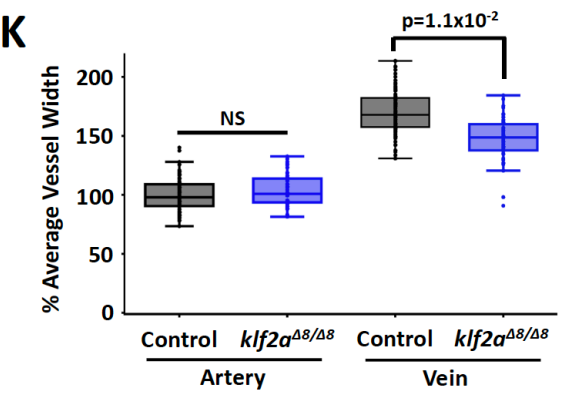

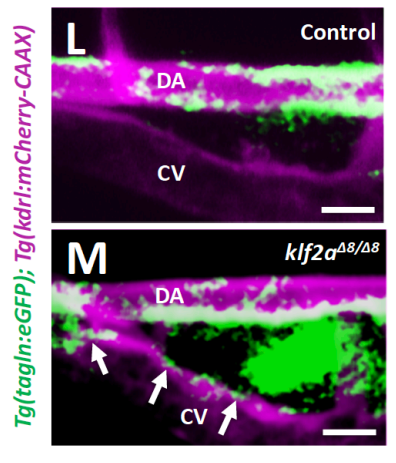




\section{Stratman, et al. Figure 7}
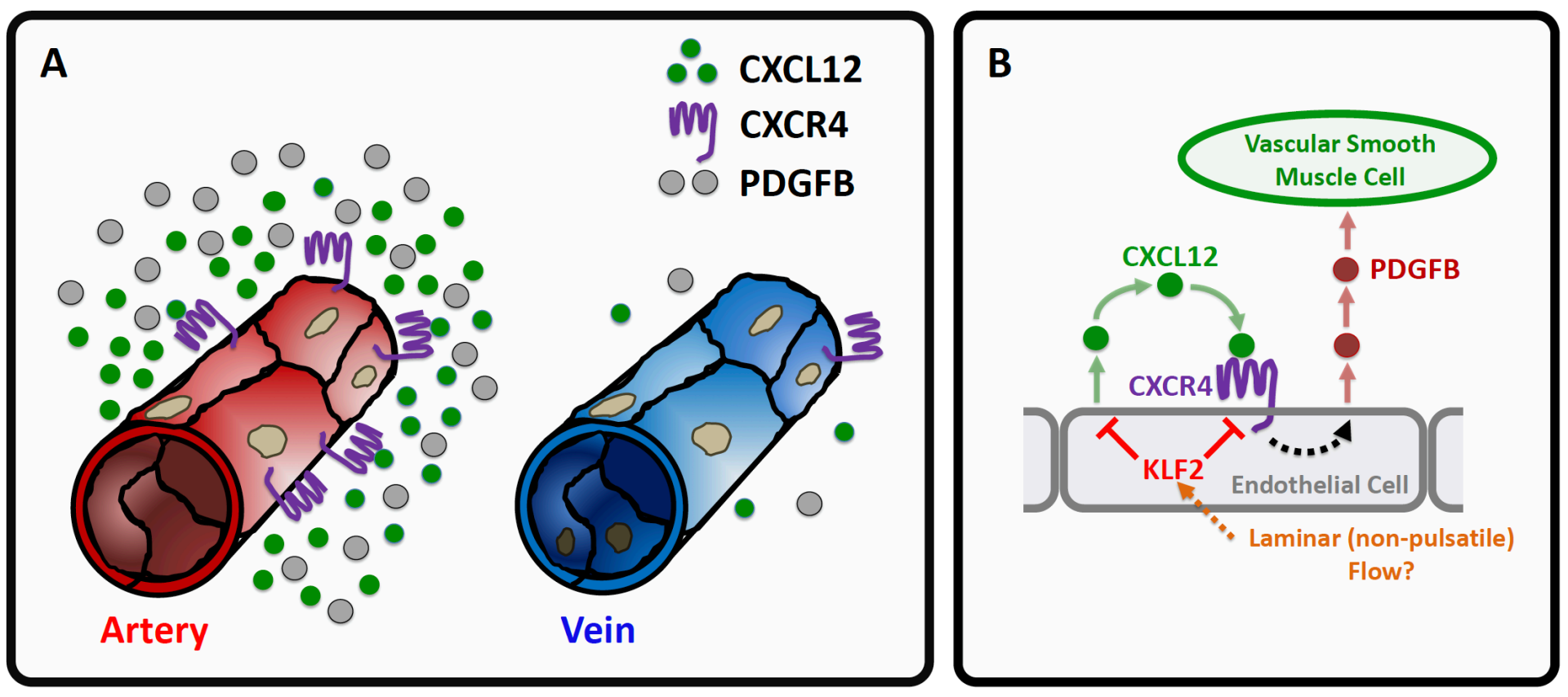
bioRxiv preprint doi: https://doi.org/10.1101/2019.12.27.889782; this version posted December 28, 2019. The copyright holder for this preprint (which was not certified by peer review) is the author/funder. All rights reserved. No reuse allowed without permission.

\section{Stratman, et al. Supplemental Figure 1}

\section{A Characterization of $c x c 112 b$ Mutant Allele}

24 bp Insertion, disrupting splicing of
Exon $1 / 2$ and generates in Frame 'STOP'

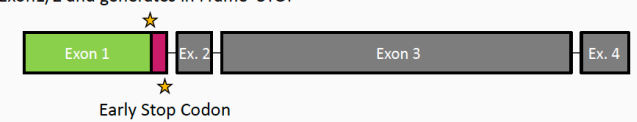

Early Stop Codon

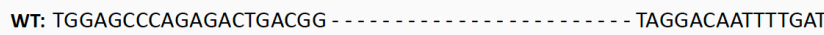
Mut: TGGAGCCCAGAGACTGACAA AAAATAGTTTGATTACATCTGCAATAGGACAATTTTGAT

\section{B}

\section{Characterization of cxcr4a Mutant Allele}

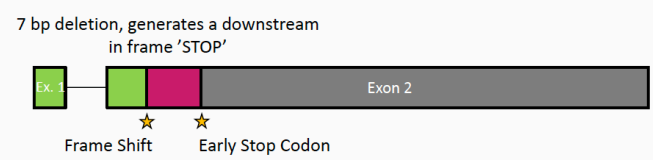

WT: CATCGGAGCCAACTTTGAGGTCCCGTGTGATGTGGAGGTCAGTCACGACTTCCAGAGG... Mut: CATCGGAGCC - . - . - . - GTGTCCCGTGTGATGTGGAGGTCAGTCACGACTTCCAGAGG...

\section{Characterization of pdgfba and pdgfb Mutant Alleles}

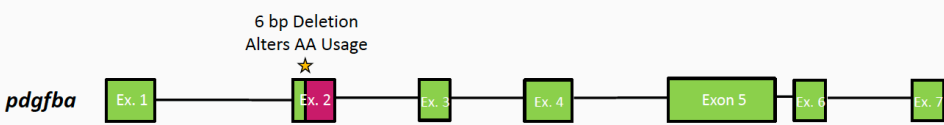

Ser Thr Ser Gly

wT: GGGGACCCTCTTCCTCC ATC TCT GGTGGTTTAGTAATGAACAGCCCAATTTCAACTGTAGACGATCTGAAG Mut: GGGGACCCTCTTCCTCT - . . . . . GGTGGTTTAGTAATGAACAGCCCAATTTCAACTGTAGACGATCTGAAG Ser Gly

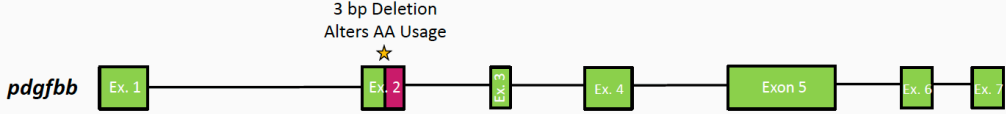

Leu His Gln

WT: CTATGGATTCAGAGAAGAGCAGAAACTGCAGGTCTTGAATTGAAGACACAGATCCAGCCTT CAC CAACTCA Mut: CTATGGATTCAGAGAAGAGCAGAAACTGCAGGTCTTGAATTGAAGACACAGATCCAGCCTT - - - CAACTCA

\section{Characterization of $k$ lf $2 a$ Mutant Allele}

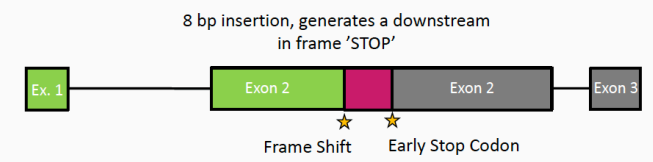

WT: TAGT - - - - - - - TACGGACCTGTACGAGGAATGGTTCCCAAAGTCAAACAAGAGGGCAACGGTGCATGCATGA Mut: GAATGGTTGTACGAGGAACCTGTACGAGGAATGGTTCCCAAAGTCAAACAAGAGGGCAACGGTGCATGCATGA 


\section{Stratman, et al. Supplemental Figure 2}

\section{HUVEC siRNA Transcript Suppression Confirmation}
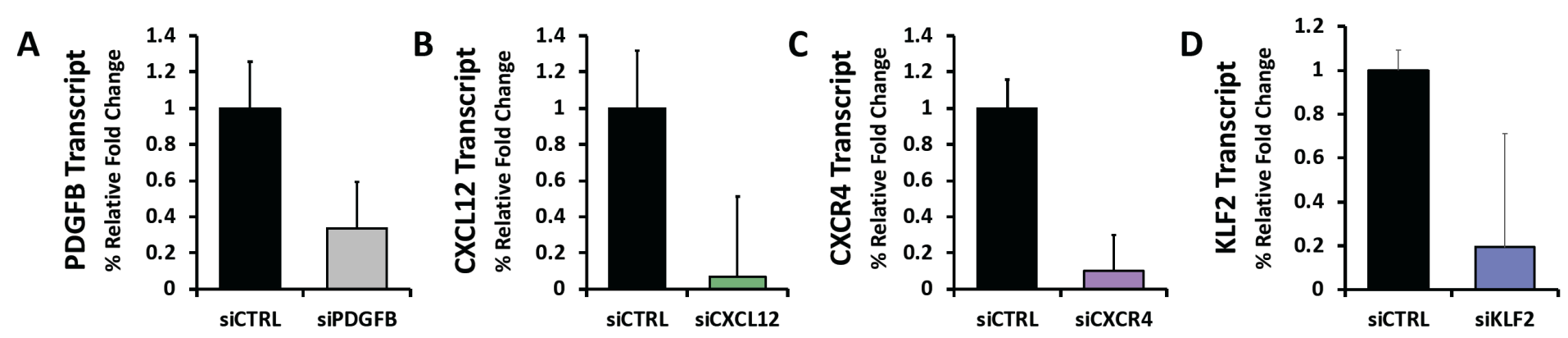